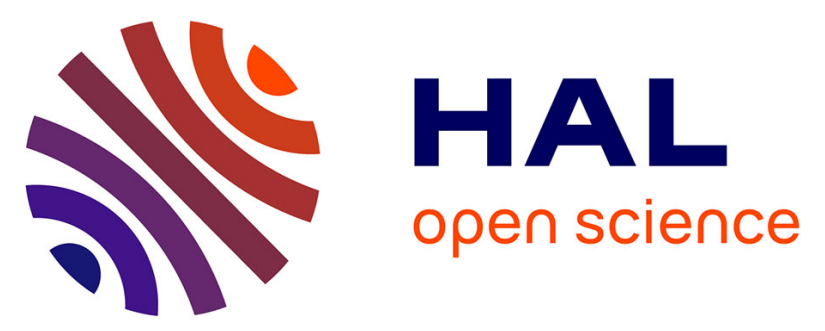

\title{
Coarse-grained elastic network modelling: A fast and stable numerical tool to characterize mesenchymal stem cells subjected to AFM nanoindentation measurements
}

L. Vaiani, E. Migliorini, E.A. A Cavalcanti-Adam, A.E. E Uva, M. Fiorentino, M. Gattullo, V.M. M Manghisi, A. Boccaccio

\section{To cite this version:}

L. Vaiani, E. Migliorini, E.A. A Cavalcanti-Adam, A.E. E Uva, M. Fiorentino, et al.. Coarse-grained elastic network modelling: A fast and stable numerical tool to characterize mesenchymal stem cells subjected to AFM nanoindentation measurements. Materials Science and Engineering: C, 2021, 121, pp.111860. 10.1016/j.msec.2020.111860 . hal-03149866

\author{
HAL Id: hal-03149866 \\ https://hal.science/hal-03149866
}

Submitted on 23 Feb 2021

HAL is a multi-disciplinary open access archive for the deposit and dissemination of scientific research documents, whether they are published or not. The documents may come from teaching and research institutions in France or abroad, or from public or private research centers.
L'archive ouverte pluridisciplinaire HAL, est destinée au dépôt et à la diffusion de documents scientifiques de niveau recherche, publiés ou non, émanant des établissements d'enseignement et de recherche français ou étrangers, des laboratoires publics ou privés. 
L. Vaiani ${ }^{1}$, E. Migliorini ${ }^{2}$, E.A. Cavalcanti-Adam ${ }^{3,4}$, A.E. Uva ${ }^{1}$, M. Fiorentino ${ }^{1}$, M. Gattullo ${ }^{1}$, V.M. Manghisi ${ }^{1}$, A. Boccaccio ${ }^{1 *}$

${ }^{1}$ Dipartimento di Meccanica, Matematica e Management, Politecnico di Bari, Bari 70126, Italy ${ }^{2}$ CNRS UMR 5628 (LMGP), Grenoble 38016, France ${ }^{3}$ Max Planck Institute for Medical Research, D-69120 Heidelberg, Germany ${ }^{4}$ Heidelberg University, D-69120 Heidelberg, Germany

*Corresponding author:

E-mail: antonio.boccaccio@poliba.it 
The knowledge of the mechanical properties is the starting point to study the mechanobiology of mesenchymal stem cells and to understand the relationships linking biophysical stimuli to the cellular differentiation process. In experimental biology, Atomic Force Microscopy (AFM) is a common technique for measuring these mechanical properties.

In this paper we present an alternative approach for extracting common mechanical parameters, such as the Young's modulus of cell components, starting from AFM nanoindentation measurements conducted on human mesenchymal stem cells. In a virtual environment, a geometrical model of a stem cell was converted in a highly deformable Coarse-Grained Elastic Network Model (CG-ENM) to reproduce the real AFM experiment and retrieve the related force-indentation curve. An ad-hoc optimization algorithm perturbed the local stiffness values of the springs, subdivided in several functional regions, until the computed force-indentation curve replicated the experimental one. After this curve matching, the extraction of global Young's moduli was performed for different stem cell samples. The algorithm was capable to distinguish the material properties of different subcellular components such as the cell cortex and the cytoskeleton. The numerical results predicted with the elastic network model were then compared to those obtained from hertzian contact theory and Finite Element Method (FEM) for the same case studies, showing an optimal agreement and a highly

51 reduced computational cost. The proposed simulation flow seems to be an accurate, fast and stable method for understanding the mechanical behavior of soft biological materials, even for subcellular levels of detail. Moreover, the elastic network modelling allows shortening the computational times to approximately $33 \%$ of the time required by a traditional FEM simulation performed using elements with size comparable to that of springs.

Keywords: Elastic Network Model, Atomic Force Microscopy, Cell Material Characterization, 


\section{1. Introduction}

Stem cells are undifferentiated cells capable of differentiation towards specific cell

61 phenotypes. The control of stem cells biology through mechanical factors remains a poorly understood topic and represents one of the main objectives of mechanobiology [1]. Mechanobiology is an interdisciplinary research area that integrates engineering and biology, exploiting the best computational and experimental techniques to investigate the interactions between external mechanical stimuli and internal adaptive response of living cells [2] and to understand the relationships linking forces acting on a cell and biophysical responses [3-5]. Computational mechanobiological models were recently developed to design biomaterials for bone tissue engineering applications [6-8].

The starting point to investigate stem cell mechanobiology is the assessment of the mechanical properties and deformation characteristics of the cell and its subcellular components. One of the most commonly adopted techniques to measure these properties is the Atomic Force Microscopy (AFM) that is widely used in experimental biology for mechanical characterization of whole living cells [911] as well as for subcellular components [12,13], and also for biological tissues in general [14-18]. It essentially consists in moving a spherical, conical or pyramidal nanoindenter fixed at the tip of a flexible cantilever of known stiffness into the surface of the material to investigate $[19,20]$. The tip displacement is detected and, based on the cantilever stiffness, is converted into force, in order to obtain a force-indentation curve that is strictly related to the stiffness of the material under examination. The effects of the AFM tip geometry and of the stress state acting on the nanoindented material were investigated in previous studies [21,22]. A common practice for assessing elastic properties of a biomaterial starting from force-indentation AFM curves exploits the hertzian contact theory $[20,23]$. The main hypotheses of this theory, in the particular case of a spherical indenter, are the following: (i) the strains in the nanoindented material are infinitesimal, (ii) the contact area is small, (iii) the nanoindented material has a linear elastic and uniform behavior, (iv) the nanoindented 
material presents a perfectly flat surface with infinite dimensions, (v) the indenter is perfectly spherical. In a real AFM tip to living cell contact not all those conditions are satisfied and is impossible to distinguish, by using this theoretical framework, the single contributions to the overall stiffness of the several subcellular components [11,24], acting as a series-system composed by different stiffness values, but only retrieve an overall cell stiffness as a weighted average of the subcellular components stiffnesses.

Nowadays computer simulations represent a powerful tool for studying physical phenomena presenting real conditions that are not reproducible in a laboratory [25]. A common numerical technique suitable for modelling deformable objects subjected to loads and constraints is the Finite Element Method (FEM) [26,27]. The main issues encountered in the finite element analysis of soft biological materials are the following: (i) the numerical convergence is problematic and often missing even for small imposed displacements due to very low Young's moduli; (ii) the large local distortions induced in the mesh and the consequent need for numerical stabilization functions; (iii) the high computational resources required for modelling complex structures. The main goal of this study is to overcome the problems related to the large deformations and distortions imposed on mesh-based models in simulations of soft biological materials. A meshless approach is therefore proposed, characterized by much more compliant topologies, that have no continuity constraints on displacement fields and related deformations [28]. Some examples of meshless modelling of living cells are presented in the review paper by Ye et al. (2016) [29] where several particle-based simulations of red blood cells interacting with a viscous fluid flow are compared. Very interesting is also the work of Vassaux and Milan (2017) [30], who studied the effect of the curvature of substrate during the adhesion of stem cells through a particle-based approach.

In this study, AFM nanoindentation measurements were first conducted on human mesenchymal stem cells. Then, a Coarse-Grained Elastic Network Modelling (CG-ENM) [31-33] was used to represent the stem cell and its subcellular components and to simulate the nanoindentation process. The pre-tensioning stress state acting on the cell due to the action of stress fibers that anchor 
110 the cell to the substrate, was also simulated and the Young's moduli of different subcellular

111 components, such as cytoskeleton and cortex, were extracted. The results obtained with the elastic 112 network model were then compared to those computed with the Hertz contact theory and the Finite 113 Element Method (FEM), showing a good agreement. We found that the proposed approach generally 114 leads to a stable numerical modelling even for large deformations and to significantly smaller 115 computational costs than those typically required in mesh-based numerical tools.

\section{2. Materials and methods}

\subsection{Study overview}

Aiming at extracting a set of global Young's moduli, a physical-mathematical scheme has been proposed (Fig. 1), implementing a reversed approach with respect to the classical characterization of new materials performed through tensile tests. In detail, the workflow includes the following steps and procedures (Fig. 1):

(i) nanoindentation experiments;

(ii) generation of a spring network model simulating the nanoindentation process of a stem cell and identification of the optimal local material properties through numerical fitting of real forceindentation curves;

(iii) transferring of the topology and of the optimized local material properties of the spring network model to a "monodimensional twin" model;

(iv) extraction of the global elastic parameters through a second computer experiment 130 simulating a common tensile test.

The last step allowed the comparison of the obtained global Young's moduli to the tabular

132 data available in the literature. Each block illustrated in Fig. 1 was extensively described in the 133 following sections. 




Fig. 1. Scheme of the workflow implemented to extract the stem cells mechanical properties. 


\subsection{AFM experiments}

For AFM nanoindentation measurements, mesenchymal stem cells (MSCs) from human primary material were used, donated by the Professor Ho group from the Department of Medicine V, Heidelberg University and isolated as described in a previous study [34].

After seeding of the mononuclear cell fraction evolving colonies were separated and MSC further expanded in plastic culture flasks. Cells were cultured in a sub-confluent monolayer in growth medium consisting of DMEM-LG supplemented with MCDB201 (40\% (v/v), Sigma), L-glutamine (2 mM, Sigma), penicillin/streptomycin (100 U/mL, Lonza), insulin transferrin selenium (1\% (v/v), Sigma), linoleic acid albumin from bovine serum albumin (1\% (v/v), Sigma), dexamethasone (10 nm, Sigma), L-ascorbic acid-2-phosphate (0.1 mM, Sigma), PDGF subunit B (PDGF-BB), epidermal growth factor (EGF) (both $10 \mathrm{ng} / \mathrm{mL}$; PreproTech, Rocky Hill, NJ, USA) and FCS (2\% (v/v), HyClone, GE Healthcare). Medium was changed twice per week and early passages 3-5 were used. MSCs were detached with Accutase (Sigma Aldrich) from the flask and plated on glass cover slips ( $24 \times 24 \mathrm{~mm}^{2}$; Menzel Gläser, Braunschweig, Germany), previously coated with $1 \mathrm{mg} / \mathrm{mL}$ of Fibronectin (Sigma) diluted in PBS. $20 \mathrm{nM}$ of bone morphogenetic protein 2 (purchased from R\&D Systems Inc. Minneapolis, MN, USA) has been added to the media to induce osteogenic differentiation of MSCs. The differentiation media was replaced once during the first week and just before the experiment.

The cell indentation experiment was realized with an AFM (Nano Wizard, JPK Instruments, Berlin) combined with an inverted optical microscope (Zeiss Axiovert 200). Glass cover slips were mounted onto a stage (BiocellTM JPK) in differentiation medium, maintaining the temperature at 37 ${ }^{\circ} \mathrm{C}$. Chromium/gold-coated cantilevers (CP-qp-SCONTBSG from sQUBE) with a colloidal probe

156 (radius $\mathrm{R}=5 \mu \mathrm{m}$ ) made of borosilicate glass were used with a nominal spring constant $k$ between $k$ $157=0.006-0.015 \mathrm{~N} / \mathrm{m}$, resonance frequency between $8-13 \mathrm{kHz}$ and length around $125 \mu \mathrm{m}$. 158 Nanoindentation was performed by lowering the AFM tip onto the cell surface. After the contact with 159 the cell, the AFM tip exerts an indentation force $F=k \cdot D$, being $D$ the cantilever deflection, which is 
registered for each position $(Z)$ taken by the piezoelectric translator. The indentation $(d)$ of the cell can hence be computed as $d=Z-D$.

The stiffness of MSCs was tested one week after osteogenic induction via osteogenic media. Indentation curves were obtained along the cell body crossing the cellular soma. The AFM was set to have a maximal indentation value no larger than $200 \mathrm{~nm}$ to obtain forces applied by the cantilever to the sample always less than $5 \mathrm{nN}$. For the calculation of the cellular mechanical properties, we averaged five curves taken at the highest point of the cell (corresponding to the soma) and we repeated the measurements on ten different mesenchymal stem cells. In detail, before computing the average value, the five curves were aligned in correspondence of the point where the AFM tip comes in contact with the cell. The function interpl available in Matlab was utilized to determine, for each experimental curve, the value of indentation force at specific indentation depths (query points). Then, the average force-indentation curve was determined, for each of the ten cells, by simply computing the average value of force in correspondence of these indentation depths and was implemented in the optimization algorithm described below in the Section 2.7.

\subsection{Geometrical modelling}

Stem cells belong to the category of eukaryotic cells and are characterized by a complex internal structure. The subcellular components that were hypothesized to be included in the stem cell model are: the nucleus, the cytoskeleton, a network of interconnected filaments [35], and the cell cortex. Stress fibers and focal adhesions, that guarantee the adhesion of the cell to the substrate where it lies, were also modelled. Focal adhesions are transmembrane receptors that establish the adhesion between the ventral side of the cell and the extracellular matrix [36-38]. Stress fibers are contractile bundles of actomyosin subjected to tensile stress, mechanically interacting with focal adhesions and additional cellular structures [39] - such as the neighboring stress fibers and the cell cortex - and exerting on them a traction force of $10 \mathrm{nN}[40,41]$. More in detail, three main types of stress fibers 
exist, distributed in different regions and characterized by different lengths, from 10 to $100 \mu \mathrm{m}$ : dorsal stress fibers, ventral stress fibers and transverse arcs [42].

The CAD model of the cell was developed in the Rhinoceros CAD software environment (Version 6, McNeel) integrated with the Grasshopper plugin. The main geometry is essentially a revolved surface (Fig. 2), with an overall size of $50 \mu \mathrm{m}$ for diameter and $13 \mu \mathrm{m}$ for height (Fig. 2c), which is consistent with the typical size of mesenchymal stem cells [43]. In order to reduce the computational effort and increase the resolution at the same time, only a quarter of the whole cell was modelled. Following previous studies $[30,44,45]$, nine stress fibers were modelled in the quarter model: three arcs, three dorsal (i.e. two entire and one comprised of two halves) and three ventral stress fibers. This quarter model includes the following components (Fig. 2b):

(i) the nucleus, represented by a sphere quarter of radius $5 \mu \mathrm{m}$, with the center placed at $6 \mu \mathrm{m}$ from the bottom surface;

(ii) 8 tensioning regions for dorsal stress fibers, 4 located in the medium-upper part of the cell cortex (representing the cell regions where the dorsal stress fibers apply traction forces in downward direction) and 4 at the bottom of the cell (representing the regions where the dorsal stress fibers are connected to the focal adhesions) (Fig. 2b);

(iii) 4 tensioning regions for transverse arcs, located in the lower part of the cell cortex and connected to the dorsal stress fibers (Fig. 2b);

(iv) 6 tensioning regions at the bottom of the cell where the ventral stress fibers are connected to the focal adhesions (Fig. 2b);

(v) the cortex region that was separated from the cytoskeleton, thus obtaining a $200 \mathrm{~nm}$ thick membrane enclosing the whole cell (Fig. 2c). The value of the cell cortex thickness used in the model is consistent with that hypothesized in previous studies [11,19];

(vi) the cytoskeleton, resulting as the volume obtained by subtracting, from the initial volume of the whole cell, the previous volumes. 


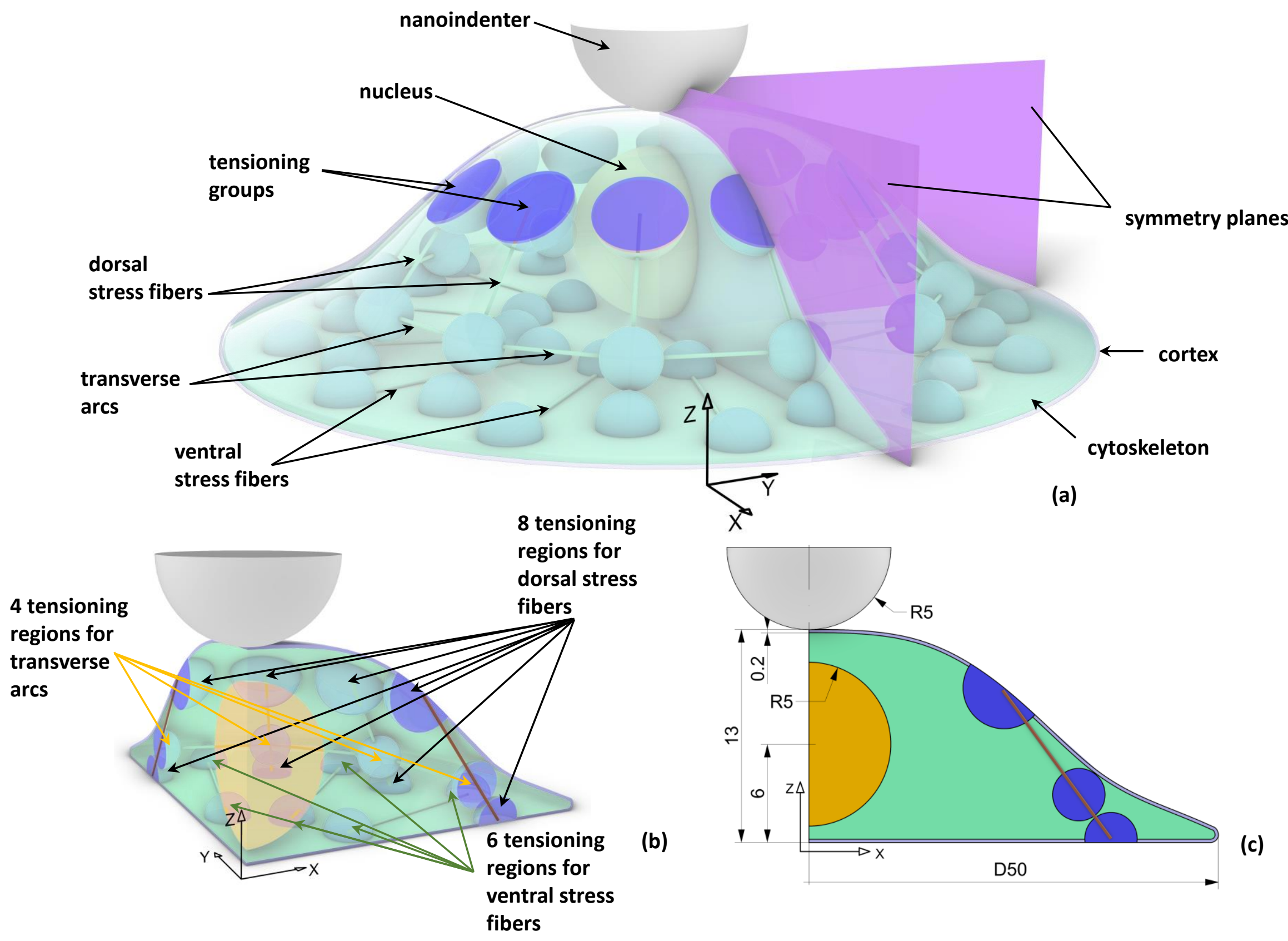

Fig. 2. Exploiting the symmetry properties of the entire cell geometry (a), just one-quarter model was considered (b). (c) Principal dimensions in micrometers of the model used in the study. 
The strategy of using tensioning regions allowed to distribute the traction force (which is a

211 relatively intense force, having the same order of magnitude as the force exerted by the AFM tip on

212 the whole cell) exerted by stress fibers on large-dimensioned regions, thus avoiding numerical issues.

213 In fact, the application of the whole traction force on a single point would produce a deformation so

214 large that any computational tool (including finite element method and coarse-grained elastic network 215 model) would inevitably undergo non-convergence problems. Tensioning regions not only allow 216 solving numerical issues, but also reproduce in a reasonably correct way the physics and the biology 217 of the cell, where internal loads do not act on point-like regions with limited dimensions but on 218 relatively wide regions, whose actual extensions are proportional to the force exerted to them [46$21948]$.

\subsection{Spring network generation}

The mathematical/physical approach used to model the stem cell, treated as a deformable object, is a coarse-grained elastic network modelling [31]. It consists of a uniform network of interconnected springs characterized by a single stiffness magnitude for the elements inside every single functional region, implementing an internal force field acting between pairs of coarse-grained lumped masses representing groups of atoms or molecules. The mutual connections between the different spring elements are spherical joints, thus enabling the maximum structural compliance, peculiar of a soft biological material.

To implement this framework, the CAD model (Fig. 3a) was exported from Rhinoceros and given in input to the open source meshing software Gmsh (version 4.5.2). A tetrahedral volumetric mesh was then generated (Fig. 3b), appropriately selecting a refined Delaunay grid algorithm as a 232 discretization rule, aiming at maximizing uniformity of spring lengths and isotropy in behavior. The average element size was set equal to $0.350 \mu \mathrm{m}$ which is a sufficiently small and adequate dimension to satisfactorily solve the problem of variable contact between the nanoindenter and the upper region of the cell cortex. 

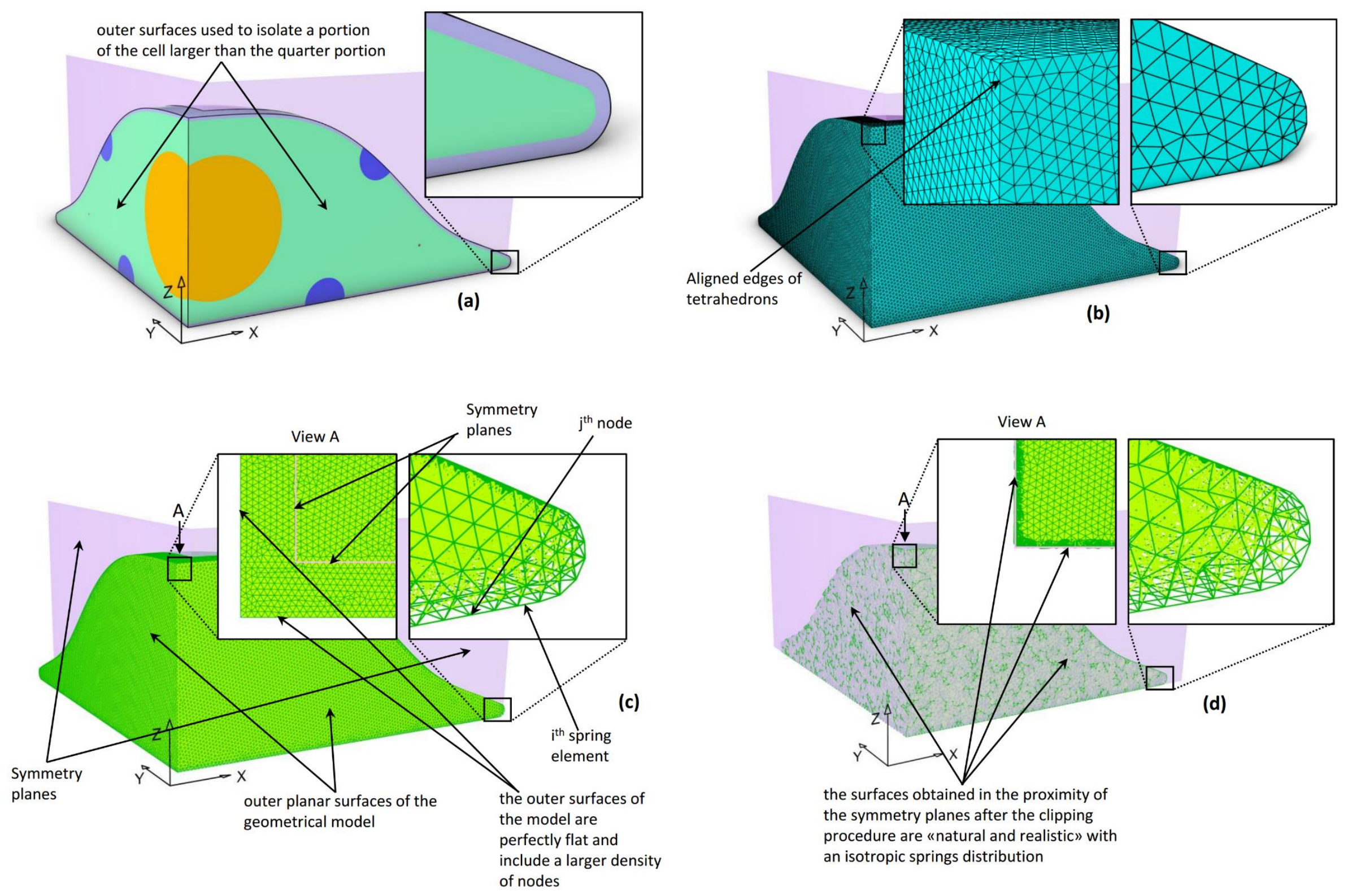

Fig. 3. The CAD model (a) generated in Rhinoceros was given in input to the open source meshing software Gmsh (version 4.5.2) where a tetrahedral volumetric mesh was generated (b). The tetrahedral mesh was then converted into a spring network model (c). To generate the quarter model, a portion of the whole cell larger than the quarter portion was initially considered (a-c). A clipping procedure was finally executed (d) to isolate the portion of the spring network model exactly corresponding to the quarter of the cell. The aligned edges of tetrahedrons shown in (b) have been obtained at the intersection of the planes delimiting the model outer surface. The clipping procedure successively carried out, allows having not aligned edges (and hence not aligned springs) on the axis along which the nanoindenter acts and consequently, an isotropic behavior of the model. 
The next operation was the transformation of the tetrahedral mesh into a spring network model

237 (Fig. 3c). To do this, we converted the edges of the tetrahedrons generated in the meshing phase into 238 springs and the vertices of the tetrahedrons into nodes where springs are interconnected. The 239 information associated to nodes position and spring connectivity was processed by an ad hoc 240 algorithm coded in the Grasshopper plugin, for the generation of the spring network into the CAD 241 environment. The result of the conversion process is a pattern of springs arranged in a tetrahedral 242 structure according to a refined Delaunay topology (Fig. 3c). The total number of springs was 887605 and the average rest length of springs was $0.345 \mu \mathrm{m}$.

To generate the quarter model, a portion of the whole cell larger than the quarter portion was initially considered (Figs. 3a-c). This portion was meshed and converted into the spring network model. A clipping procedure was finally executed (Fig. 3d) to isolate the portion of the spring network exactly corresponding to the quarter of the cell. With this strategy, we solved two main problems that arise when the quarter model is built directly using cutting planes exactly corresponding to the symmetry planes of the cell. The first problem is related to the isotropy of the model: the refined

Delaunay algorithm, selected to build the tetrahedral mesh, populates the outer planar surfaces of the geometrical model with a quite regular triangular tessellation and so the density of nodes on these surfaces will be higher than that of any other plane crossing the model. This implies that if the outer planes of the geometrical model correspond to the symmetry planes, on those planes a larger density of nodes and hence a larger density of springs will result, and also this local distribution of springs will be perfectly planar, which contrasts the hypothesis of isotropy of the spring network. The second problem is related to the structural response of the nanoindented material. If the outer planes of the geometrical model correspond to the symmetry planes of the cell, at the intersection between the two planes, the refined Delaunay algorithm will build tetrahedrons with one of the edges aligned to the central axis around which the revolution of the cell model takes place. These edges will be converted into springs and due to their alignment with the central axis, which is also the direction along which the nanoindenter acts, the structural response of the cell model to the nanoindentation process will 
mostly depend on those aligned springs. However, this contrasts the hypothesis of isotropic mechanical behavior of the model. The above-mentioned problems are overcome and solved with the described clipping procedures that allow, definitely, leaving a quarter of the cell with an isotropic and a more "natural and realistic" springs distribution, especially in the regions close to the symmetry planes.

\subsection{Definition of spring groups}

The next phase was the definition, into the CAD environment, of different spring groups functional to the subsequent simulation (Fig. 4). The spring groups that were defined are the following. (i) Nucleus - the central spherical region characterized by higher stiffness compared with that of the cytoskeleton. (ii) Tensioning groups - the regions of the cytoskeleton pulled downwards or upwards (in case of dorsal stress fibers) or pulled together (in case of transverse arcs). (iii) Cortex - the external surface surrounding the whole cell, characterized by a constant thickness. (iv) Base the lowest layer of the cell, representing the flat surface adherent to a substrate. (v) Contact - the portion of the cell cortex that enters in contact with the spherical indenter. (vi) Free - the remaining unconstrained region mainly corresponding to the cytoskeleton, affected by the boundary conditions imposed by adjacent groups, always interconnected to it.

\subsection{Simulation}

All the geometrical entities composing the spring network model of the cell were then imported in Abaqus (version 6.14, Dassault Systèmes) where a spherical nanoindenter with a $5 \mu \mathrm{m}$ radius - which is the same radius of the nanoindenter used in the experiments - was created and modelled as a rigid part aligned to the revolution axis of the cell model.

The springs were modelled as truss elements, i.e. two-node elastic rods with a constant cross section $A$, set equal to $1 \mu \mathrm{m}^{2}$, and a rest length $L_{0}$ equal to the average dimension of the edges of the 


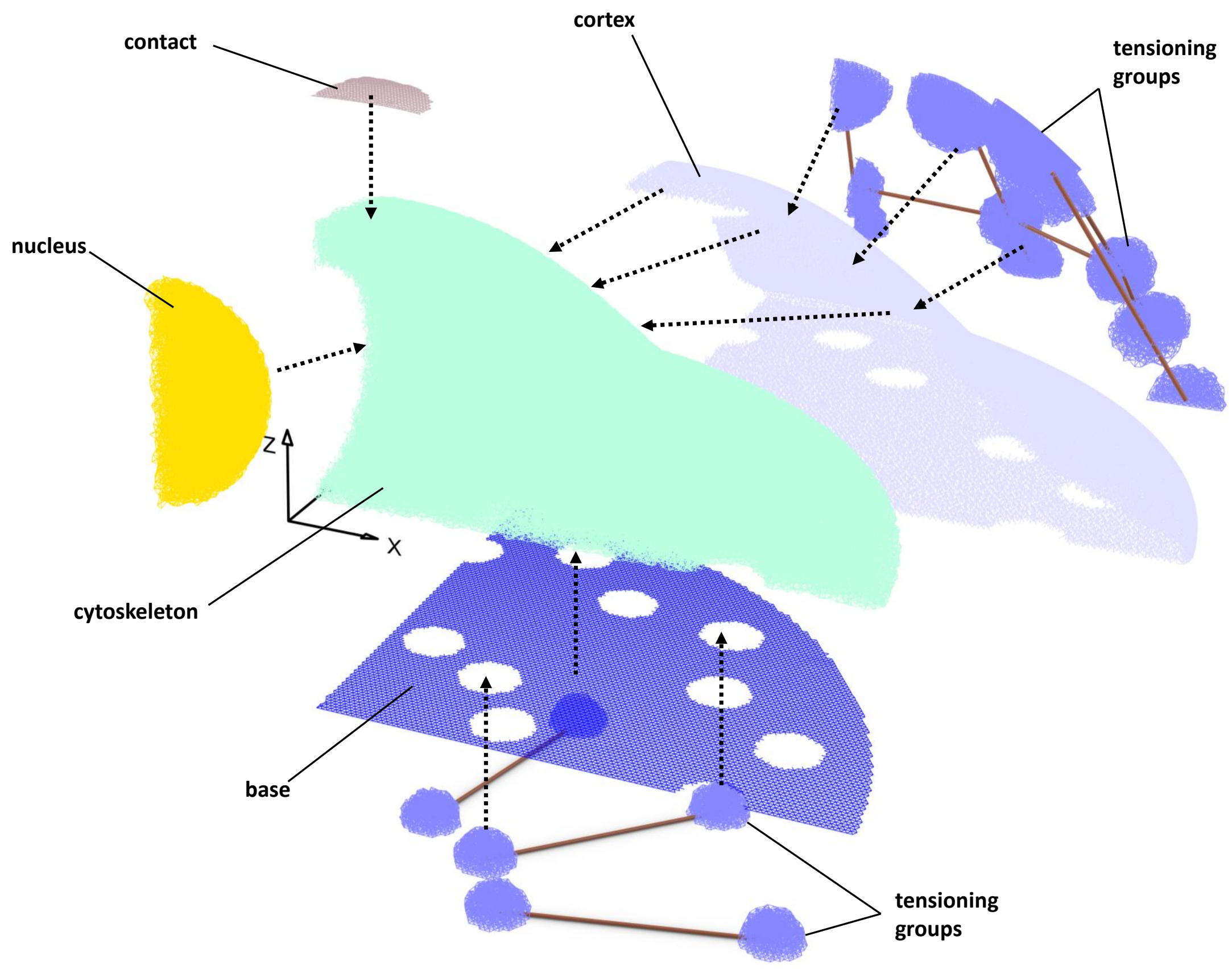

Fig. 4. Spring groups defined in the model. 
only along the axis connecting the nodes. As the truss elements are connected one each other with

289 spherical joints, that constraint only the relative positions, leaving free the relative rotations, no bending stiffness has been defined for this kind of elements. The extensional spring stiffness $E_{l o c} A / L_{0}$ was defined by setting a local Young's modulus, one for cytoskeleton springs $E_{l o c, c y t}$ and one for cortex springs $E_{l o c, c o r}$, assuming for the nucleus region a local modulus $E_{l o c, n u c}$ one order of magnitude higher than the average stiffness of the cytoskeleton. $E_{l o c, c o r}$ and $E_{l o c, c y t}$ were determined via the optimization algorithm outlined in the next section, that iteratively compares the numerical forceindentation curve computed in the simulation with the same curve obtained in the nanoindentation measurements previously described. The choice of setting the value of the material properties for the nucleus and not to optimize it derives from the fact that, being the nucleus rather distant from the nanoindenter tip, the system is not sufficiently sensitive to "capture" the structural response of the nucleus and therefore to analyze its mechanical behavior. The same hypothesis was followed in a previous study [11].

The contact between the nanoindenter and the upper region of the cell was assumed as frictionless and was solved by implementing the "hard contact" algorithm available in Abaqus. on a physical-mathematical level as incremental static problems, thus excluding all dynamic contributions (inertia, time-dependent effects, dissipations, etc.). The condition of "nonlinear geometry" was enabled in every simulation, in order to correctly map the large local deformation field in the contact region.

The boundary and loading conditions acting on the model were the following (Fig. 5). (i) No 310 displacement or rotation allowed for the base layer of the cell (encastre) for reproducing adhesion. 311 (ii) $10 \mathrm{nN}[40,41,46]$ forces imposed on every single tensioning group for stress fibers (for the 312 tensioning groups crossing the symmetry planes a $5 \mathrm{nN}$ force was applied in order to achieve a $10 \mathrm{nN}$ 313 force when mirroring the quarter model). The overall force in every group was distributed as $25 \%$ in 


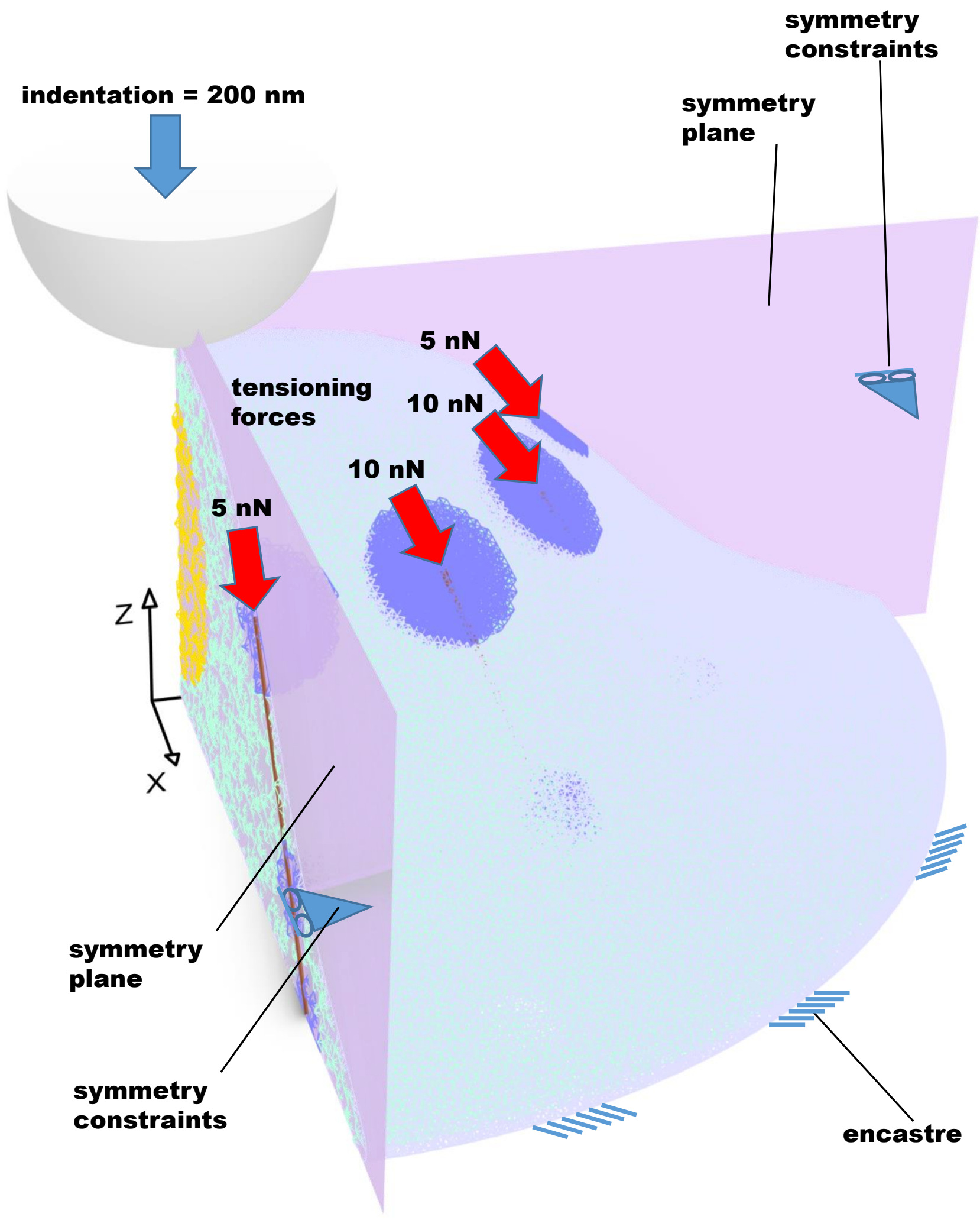

Fig. 5. Boundary and loading conditions imposed to the cell in the simulation environment. For the sake of clarity only the forces acting on the visible tensioning groups are indicated and not those acting on the internal ones. 
an outer shell and $75 \%$ in an inner half-sphere, in order to realize a sort of force gradient when moving

315 from the highly-tensioned central region to the unloaded free elements neighboring the tensioning groups [46]. In order to implement such a condition, the force per node in the inner half-sphere region

$317 \mathrm{~F}_{1, \text { inn }}$ was set equal to $2 \mathrm{~F}_{1, \text { out }}$, the latter being the force per node for the nodes in the outer shell region 318 (Fig. 6). With this strategy, a non-natural and non-physical sudden step-shaped magnitude change of 319 the force acting on the tensioning groups was avoided, thus realizing a gradient for the force per node 320 [46-48]. (iii) A $200 \mathrm{~nm}$ displacement imposed to the rigid spherical indenter in the second step. (iv) 321 Symmetry constraints imposed on the symmetry planes delimiting the model. forces; this combination has proved to be optimal to ensure the stability of the solver, thus avoiding under/overflow issues.

\subsection{Optimization}

An optimization algorithm was written in Matlab environment for iteratively comparing the

force-indentation curves obtained as experimental AFM reports to those predicted in the numerical simulations (Fig. 7). In detail, in the optimization algorithm, the fmincon tool available in the optimization toolbox of Matlab, was implemented, that is devoted to find the minimum of constrained 331 nonlinear multivariable functions. Starting from guess values for $E_{l o c, c o r}$ and $E_{l o c, c y t}$, defining the local spring stiffnesses for cortex and cytoskeleton respectively and perturbing them, different resulting curves were obtained as output from the numerical solver. Following a previous study [12], for each cell sample, optimization runs were started from five different sets of material properties previously randomly generated. Thanks to this strategy and to the large range of variability chosen for material 336 parameters, the whole search space was covered, thus increasing the probability of finding the global optimum. The algorithm iterated this cycle until the least squared difference between experimental and numerical force-indentation curves was less than a pre-defined numerical threshold. The optimal

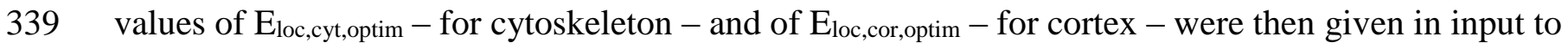




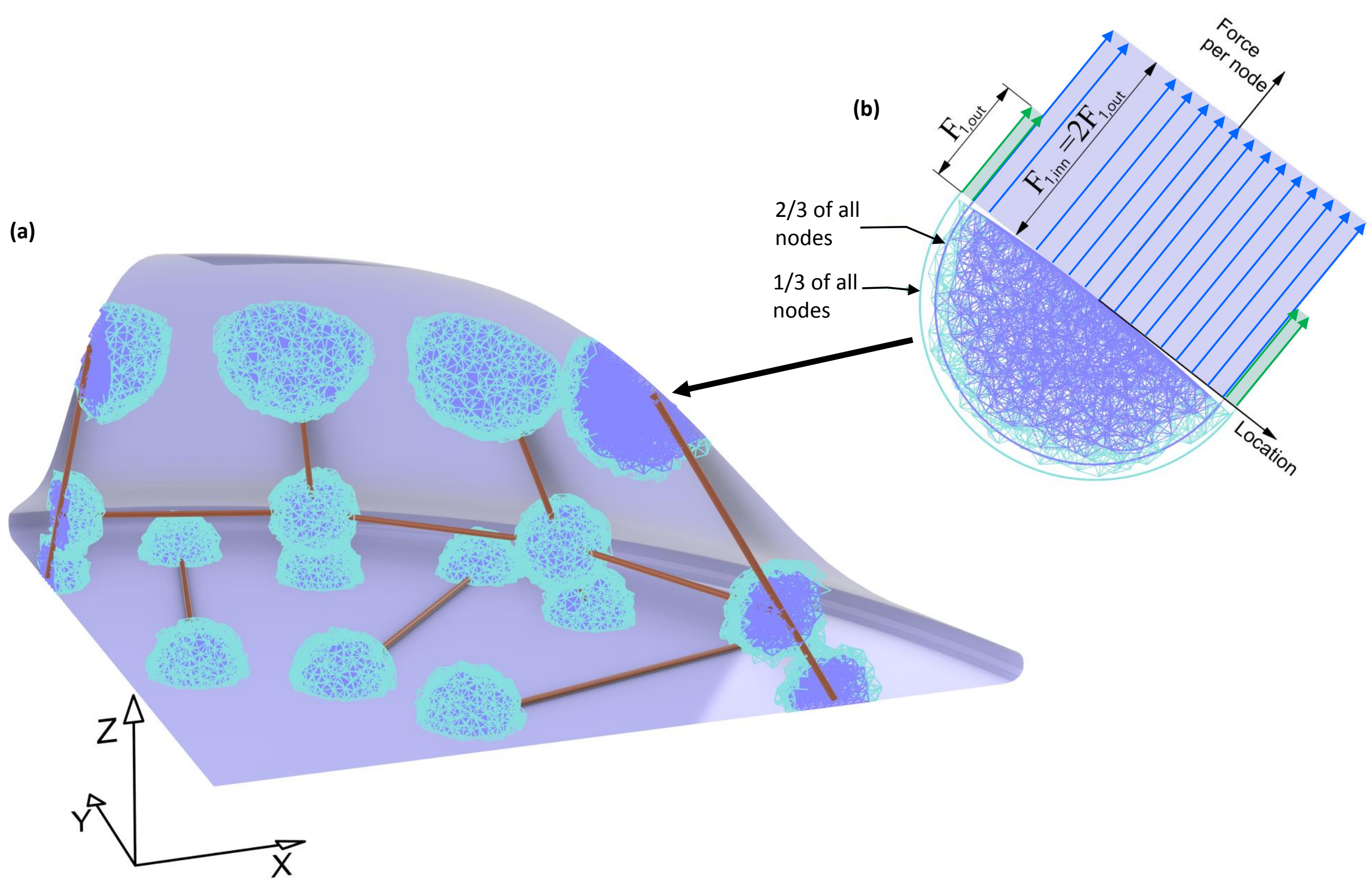

Fig. 6. The overall force in every group (a) was distributed as $25 \%$ in an outer shell and $75 \%$ in an inner half-sphere (b), in order to realize a sort of force gradient when moving from the highly-tensioned central region to the unloaded free elements neighboring the tensioning group. 


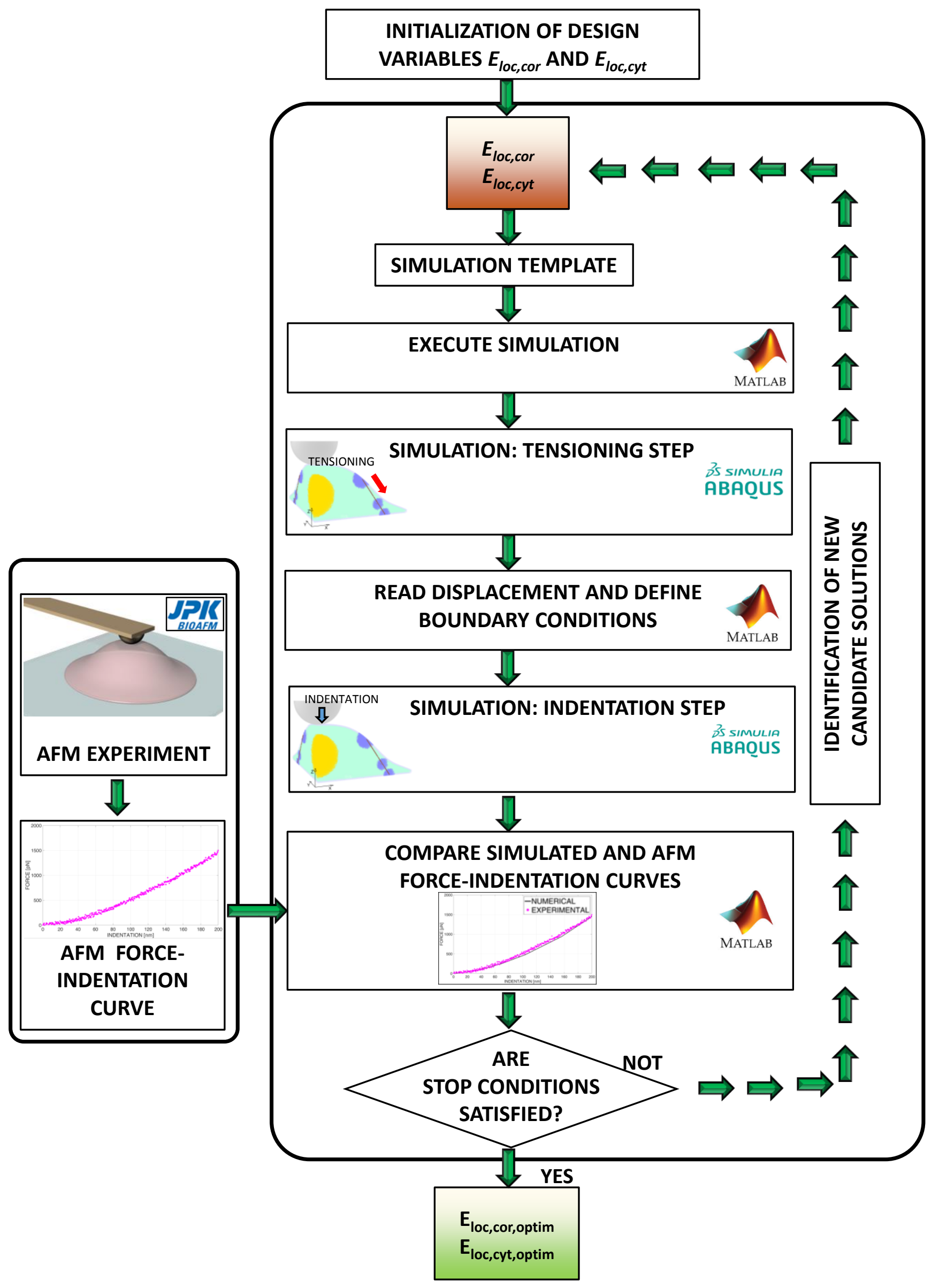

Fig. 7. Scheme of the optimization algorithm implemented to determine the optimal local material properties for cytoskeleton and cortex. 
a "monodimensional twin" model (sect. 2.8), that aimed at converting them in global Young's moduli,

341 comparable with those presented in classical engineering and scientific literature for materials.

The optimization flow above described was executed to calculate the elastic parameters

$E_{\text {loc,cyt,optim }}$ and $E_{\text {loc,cor,optim }}$ for ten different stem cell samples. In detail, for each nanoindented cell

sample, five curves were experimentally retrieved by measuring force and displacement on the rigid nanoindenter. The optimization algorithm utilized as a reference experimental curve for each investigated stem cell sample, the curve averaged over the five measurements carried out on each single cell.

\subsection{Extraction of mechanical properties from the monodimensional twin model}

The same procedure previously described to build the model of the cell was replicated for a "monodimensional twin" model, i.e. a cylinder of approximately the same volume of the cell, presenting an overall height $H_{I D T}=70 \mu \mathrm{m}$, a diameter $D_{I D T}=14 \mu \mathrm{m}$ and characterized, according to the UNI EN 10002-1 standard (Metallic materials - Tensile Testing) by an aspect ratio of height : diameter $=5: 1$. This cylinder was utilized to extract the global Young's moduli of cytoskeleton and cortex (Fig. 8). This second model presented the same geometrical (same spring network topology, cross sections and rest lengths) and structural (same local spring stiffnesses) characteristics of the cell. Also in this case, exploiting the symmetry of the problem, just one-quarter of the cylindrical volume was modelled and symmetry constraints were applied to the nodes lying on the symmetry planes. As in the cell model, outer planes of the geometrical model different from the symmetry planes were utilized to isolate a portion of the cylindrical volume larger than the quarter portion.

361 Successively, a clipping procedure was utilized to isolate the portion of the spring network exactly delimited by the symmetry planes (Fig. 8). With this strategy, we guaranteed the isotropic structural response of the monodimensional twin model.

For each of the ten stem cell samples, two optimal values were determined through the 

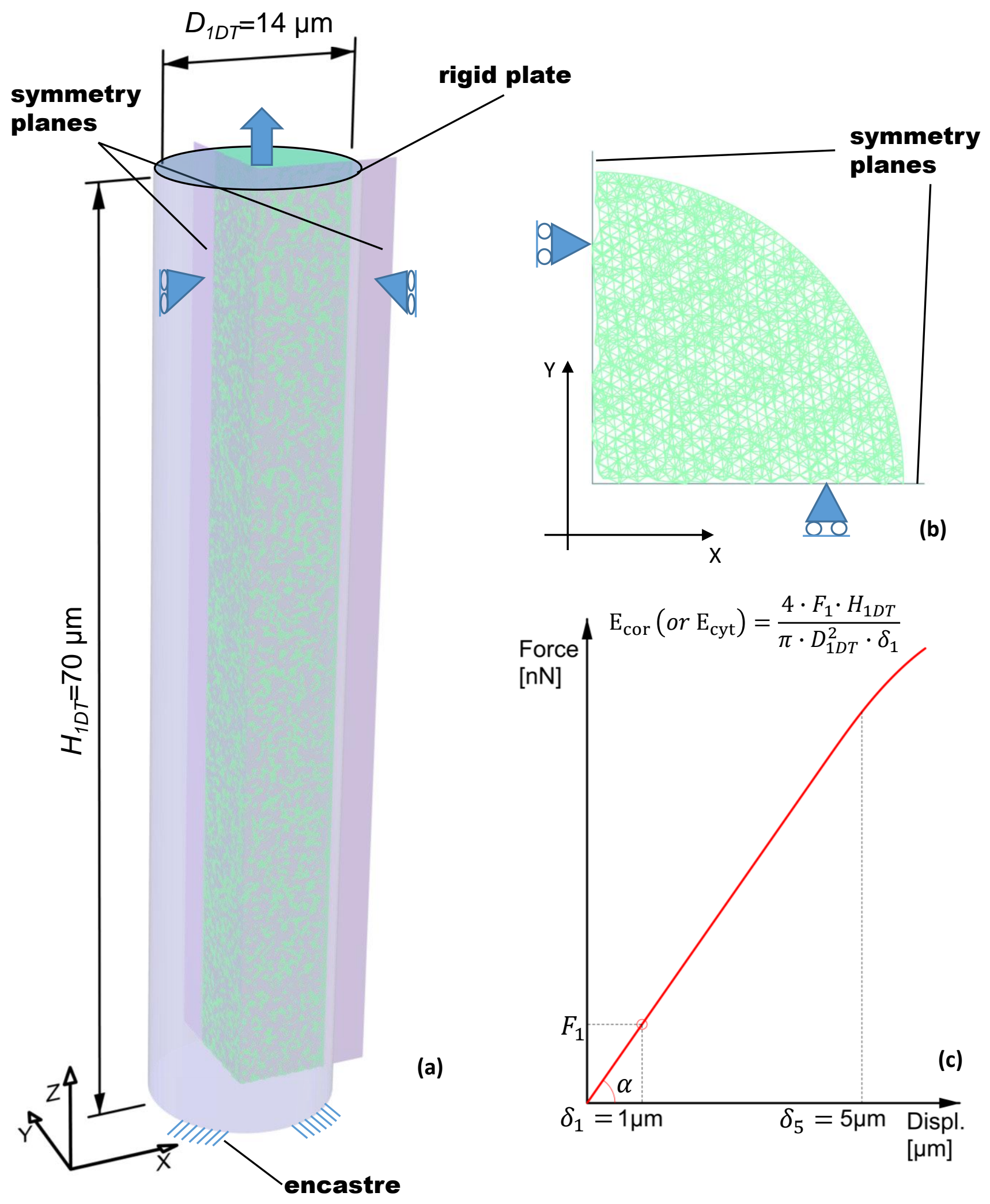

Fig. 8. (a) Simulation setup for the monodimensional twin model (dimensions in micrometers); (b) Magnified cross sectional view of the quarter model; c) Procedure for the extraction of global Young's moduli. 
for the cortex. These quantities were then used for defining the stiffness of the springs constituting

367 the monodimensional twin model and hence a virtual tensile test to extract a typical force368 displacement curve was performed. In the simulations, constraints preventing all the displacements 369 were imposed to the nodes lying on a flat base of the cylinder, while a displacement of $\delta_{5}=5 \mu \mathrm{m}$ was 370 imposed to the nodes lying on the other flat base via a rigid plate that was utilized to easily retrieve 371 the value of the reaction force. Therefore, for each cell sample, two force-displacement curves were 372 traced, one was obtained by assigning to the springs the Young's modulus Eloc,cyt,optim (for the 373 cytoskeleton), the other by assigning the Young's modulus Eloc,cor,optim (for the cortex). As in the common experimental practice, the slope of the curve was extracted for the calculation of the resulting global Young's moduli $\mathrm{E}_{\mathrm{cor}}$ and $\mathrm{E}_{\mathrm{cyt}}$, for cortex and for cytoskeleton, respectively. The value of force $F_{1}$ registered for the displacement $\delta_{1}=1 \mu \mathrm{m}$ was utilized to determine the global Young's modulus as (Fig. 8).

$$
\mathrm{E}_{\mathrm{cor}}\left(\text { or } \mathrm{E}_{\mathrm{cyt}}\right)=\frac{4 \cdot F_{1} \cdot H_{1 D T}}{\pi \cdot D_{1 D T}^{2} \cdot \delta_{1}}
$$

\section{Results and Discussion}

The average experimental force-indentation curves were compared to those predicted numerically (as described in Sect. 2.7) and the optimization algorithm changed many times the elastic parameters of the spring network model of the stem cell, until the difference between the two curves was minimized (Table 1). The rather high values of the correlation coefficient $R^{2}$, used as a metric for the detail level of the curve fitting, could be considered as a proof of the reasonably good adequacy of the model to reproduce the physics of the problem (Fig. 9, Table 2). Error bars were also superimposed to the average experimental force-indentation curves to show the dispersion of data at different indentation depths (Fig. 9).

Through the procedure described in Sect. 2.8, the global Young's moduli for cortex and cytoskeleton were then extracted from the monodimensional twin models and compared to those 

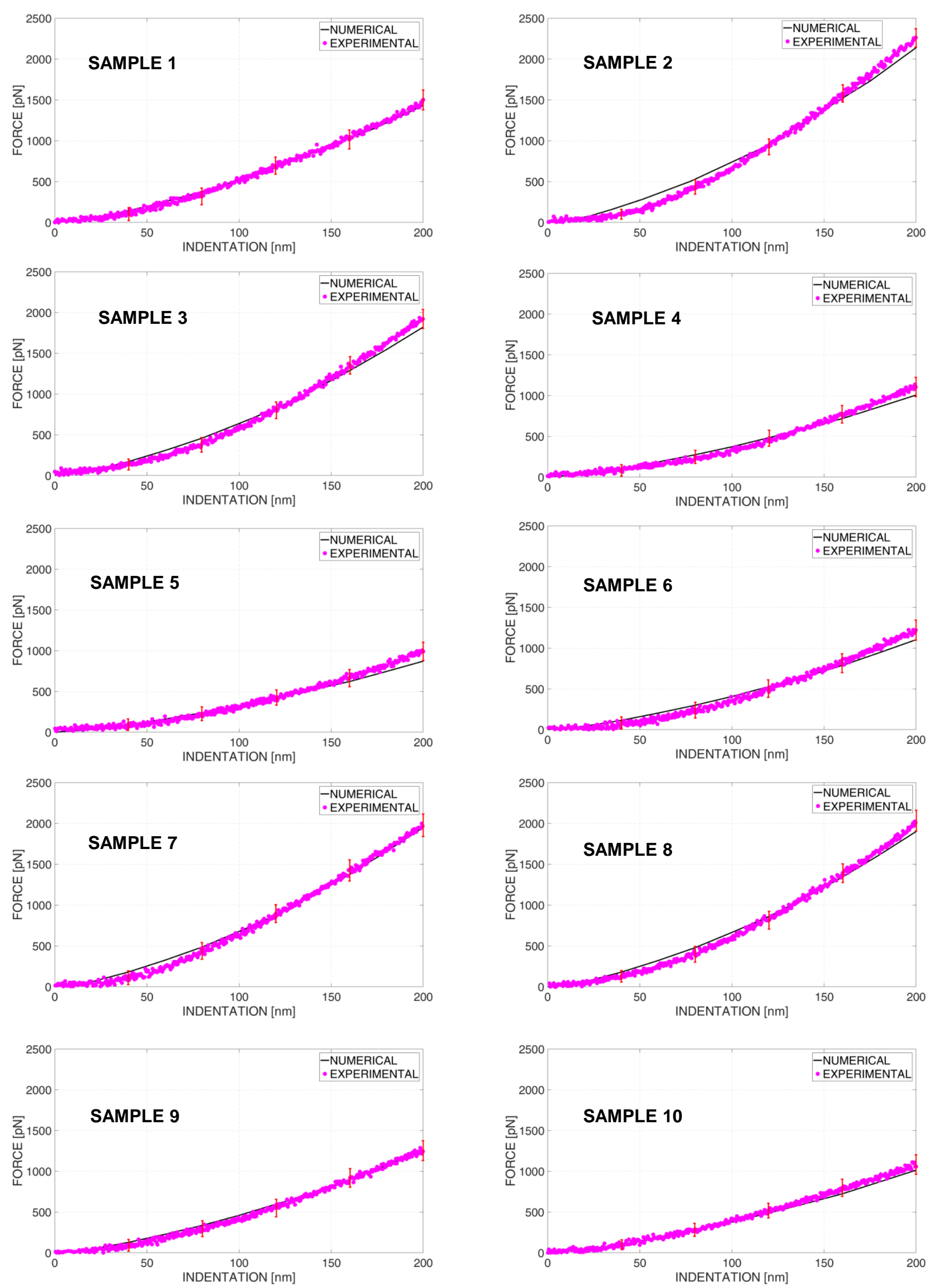

Fig. 9. Experimental force-indentation curves registered for the ten stem cell samples and related numerical curves predicted via the optimization algorithm. Error bars were superimposed to the average experimental force-indentation curves to show the dispersion of data at different indentation depths. 
391 calculated in a previous study [24] implementing both a FEM model based on the Arruda-Boyce

392 hyperelastic constitutive law [23,49] and an analytical model based on the Hertz contact theory [50] 393 (Table 3).

394 Table 1. Optimal local material properties predicted via the optimization algorithm.

\begin{tabular}{c||cc}
\hline & \multicolumn{2}{|c}{ Coarse-Grained Elastic Network } \\
\hline Sample N. & Eloc,cyt,optim [Pa] & Eloc,cor,optim [Pa] \\
\hline Sample 1 & 160 & 508 \\
Sample 2 & 252 & 490 \\
Sample 3 & 208 & 504 \\
Sample 4 & 110 & 470 \\
Sample 5 & 95 & 415 \\
Sample 6 & 127 & 485 \\
Sample 7 & 230 & 480 \\
Sample 8 & 221 & 507 \\
Sample 9 & 140 & 490 \\
Sample 10 & 120 & 470 \\
\hline
\end{tabular}

Table 2. Correlation coefficient $R^{2}$ values related to the experimental-numerical curve fitting,

397 computed for the proposed coarse-grained elastic network model versus the same coefficient values computed for the FEM model [24].

\begin{tabular}{c||c||c}
\hline \multicolumn{1}{c||}{} & \multicolumn{2}{c}{$R^{2}$} \\
\hline Sample N. & $\begin{array}{c}\text { Coarse-Grained } \\
\text { Elastic Network }\end{array}$ & FEM \\
\hline Sample 1 & 0,9962 & 0,9825 \\
Sample 2 & 0,9836 & 0,9979 \\
Sample 3 & 0,9862 & 0,9984 \\
Sample 4 & 0,9776 & 0,9920 \\
Sample 5 & 0,9733 & 0,9947 \\
Sample 6 & 0,9746 & 0,9959 \\
Sample 7 & 0,9929 & 0,9936 \\
Sample 8 & 0,9893 & 0,9963 \\
Sample 9 & 0,9952 & 0,9833 \\
Sample 10 & 0,9949 & 0,9737 \\
\hline
\end{tabular}


Table 3. Global material properties computed with the proposed coarse-grained elastic network

401 model compared to those predicted via the FEM method and the Hertz contact theory [24] (applied to the experimental force-indentation data).

\begin{tabular}{c||cc||cc||c}
\hline \multicolumn{1}{l||}{} & \multicolumn{2}{|c||}{$\begin{array}{c}\text { Coarse-Grained } \\
\text { Elastic Network }\end{array}$} & \multicolumn{2}{|c||}{$\begin{array}{c}\text { Finite Element Method, } \\
\text { Arruda-Boyce }\end{array}$} & $\begin{array}{c}\text { Hertzian Contact } \\
\text { Theory }\end{array}$ \\
\hline Sample N. & $E_{\text {cyt }}[\mathrm{Pa}]$ & $\mathrm{E}_{\text {cor }}[\mathrm{Pa}]$ & $\mathrm{E}_{\text {cyt }}[\mathrm{Pa}]$ & $\mathrm{E}_{\text {cor }}[\mathrm{Pa}]$ & E \\
\hline Sample 1 & 2911 & 9216 & 3519 & 9754 & 4110 \\
Sample 2 & 4578 & 8898 & 5290 & 9687 & 6350 \\
Sample 3 & 3771 & 9144 & 4462 & 9671 & 5200 \\
Sample 4 & 1999 & 8352 & 2575 & 9738 & 3195 \\
Sample 5 & 1722 & 7531 & 2258 & 9645 & 2800 \\
Sample 6 & 2286 & 8751 & 2901 & 9570 & 3410 \\
Sample 7 & 4152 & 8666 & 4638 & 9807 & 5550 \\
Sample 8 & 3988 & 9161 & 4551 & 9729 & 5680 \\
Sample 9 & 2527 & 8845 & 3129 & 9555 & 3455 \\
Sample 10 & 2166 & 8484 & 2640 & 9618 & 3050 \\
\hline
\end{tabular}

403

In detail, the values of $E_{c y t}$ and $E_{c o r}$ predicted through the Finite Element Method and listed in Table 3, were computed starting from the optimal hyperelastic parameters proper of the Arruda-

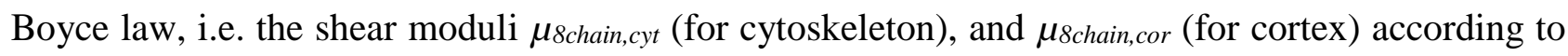
the following relationships: $\mathrm{E}_{\mathrm{cor}}=2(1+v) \mu_{\text {8chain,cor, }} \mathrm{E}_{\mathrm{cyt}}=2(1+v) \mu_{8 \text { chain,cyt }}$ where $v$, the Poisson's ratio, was set equal to 0.4999 to account for material incompressibility $[12,19]$.

The proposed coarse-grained elastic network model has some limitations. First, the Poisson's ratio is a non-controllable parameter [33], which directly depends on the discretization rule of the deformable volume, consisting in a nearly-regular and hence isotropic network of interconnected tetrahedrons. Second, the organelles residing into the cytoplasm were considered as a part of it and their material properties were "confused" with those of cytoskeleton. Third, even if the proposed model is capable of distinguishing, unlike the hertzian contact theory, the material properties of different subcellular components, in the current study a clear and direct experimental demonstration of the correctness of the model predictions is missing. Ideally, to demonstrate this, nanoindentation 
measurements should first be carried out on cells, the proposed coarse-grained elastic network model

418 should then be implemented to determine the material properties of different subcellular components.

419 At this point, the different subcellular components should be physically isolated, nanoindented and analyzed, one by one, with the proposed model. If the model works correctly, the material properties predicted before and after the isolation should be the same. An intriguing procedure recently proposed [51] that can be adopted to separate the single contributions may consist in treating pharmacologically and selectively the single subcellular component. One should first nanoindent the cell and retrieve the force-indentation curve. Based on this curve, the proposed model should be implemented to determine the mechanical properties of the different subcellular components. At this point, a pharmacological treatment can be made to alter the mechanical properties of a specific subcellular component. Obviously, this leads to the alteration of the "global" force-indentation curve. We expect that by implementing the proposed framework to the altered force-indentation curve, the system will predict altered mechanical properties for the pharmacologically targeted component and the same mechanical properties - as those predicted in the cell before the pharmacological treatment - for the components not targeted. However, due to compensatory effect inside the cell, we believe that the selectivity of the pharmacological treatment could be an issue to investigate. For instance, the chromatin condensation in the nucleus with Trichostatin A (TSA) has been targeted in a previous work but that induced an unpredictable change in the stiffness of the nucleus that is cell-dependent and a slight stiffening of the cytoplasm as compensatory effect [51]. In the future, it will be interesting to address these aspects by engineering a synthetic eukaryote cell with a bottom-up approach and therefore isolating the influence of each component on the mechanical properties of the engineered 438 cell, as a result of a multi-variable experimental plan. Fourth, hypothesizing to schematize the cell as 439 a revolved surface, just one-quarter model was considered and symmetry constraints were applied on 440 the symmetry planes. Indeed, symmetry constraints can give edge effects especially in the case where 441 the extension of the constrained region is comparable to the extension of the unconstrained region. 442 However, in the proposed model, the extension of the constrained region is much smaller than that of 
the unconstrained region. Just to give an idea of this, we can state that the number of nodes where

444 symmetry constraints act is the $5.97 \%$ of the total number of nodes. Preliminary analyses revealed 445 that the generic force-indentation curve computed with the one-quarter model is practically overlapped to that predicted with the entire model. For instance, implementing $\mathrm{E}_{\mathrm{cyt}}$ and $\mathrm{E}_{\mathrm{cor}}$ computed for the cell sample 9 we found, for a $200 \mathrm{~nm}$ indentation depth, that the force predicted with the entire model differ from that predicted with the quarter model by less than $0.6 \%$. This consideration allows us to conclude that the edge effects related to the use of symmetry constraints are negligible and hence the predictions of the one-quarter model are overlapping to those obtained with the entire model.

However, in spite of these limitations, the predicted displacement fields appear consistent with the nanoindenter geometry and with the physics of the problem (Fig. 10 (a)) as well as the predictions of the proposed model are consistent with the experimental results reported in the literature. The predicted material properties, in fact, are in good agreement with those measured via atomic force microscopy and Hertz contact theory in other studies [52-54]. Interestingly, the material properties predicted through the coarse-grained elastic network model are consistent with those computed via the Finite Element Method and via the Hertz contact theory reported in a previous study [24]. It is also interesting to observe, and is a further proof of the correctness of the model predictions, that the values of the Young's modulus $E_{\text {Hertz }}$ computed with the Hertz contact theory falls - for each of the ten cell samples investigated and for both, coarse-grained elastic network model and FEM - within the interval $\left[\mathrm{E}_{\mathrm{cyt}} ; \mathrm{E}_{\mathrm{cor}}\right]$, and are closer to $\mathrm{E}_{\mathrm{cyt}}$. This result is consistent with the physics of the problem. Given the prevalence of the cytoskeleton volume with respect to the cortex volume, it is reasonably correct that the average material properties predicted by the coarse-grained elastic network model are closer to those of the most prevalent material. In order to make a coherent statistical comparison between the sets of Young's moduli obtained with the coarse-grained elastic network model and with the finite element method, to the values computed via the Hertz contact theory, an equivalent Young's modulus must be defined for the former cases. To this purpose, the following semi-quantitative procedure is therefore proposed. The action of the AFM tip on the nanoindented cell can be 


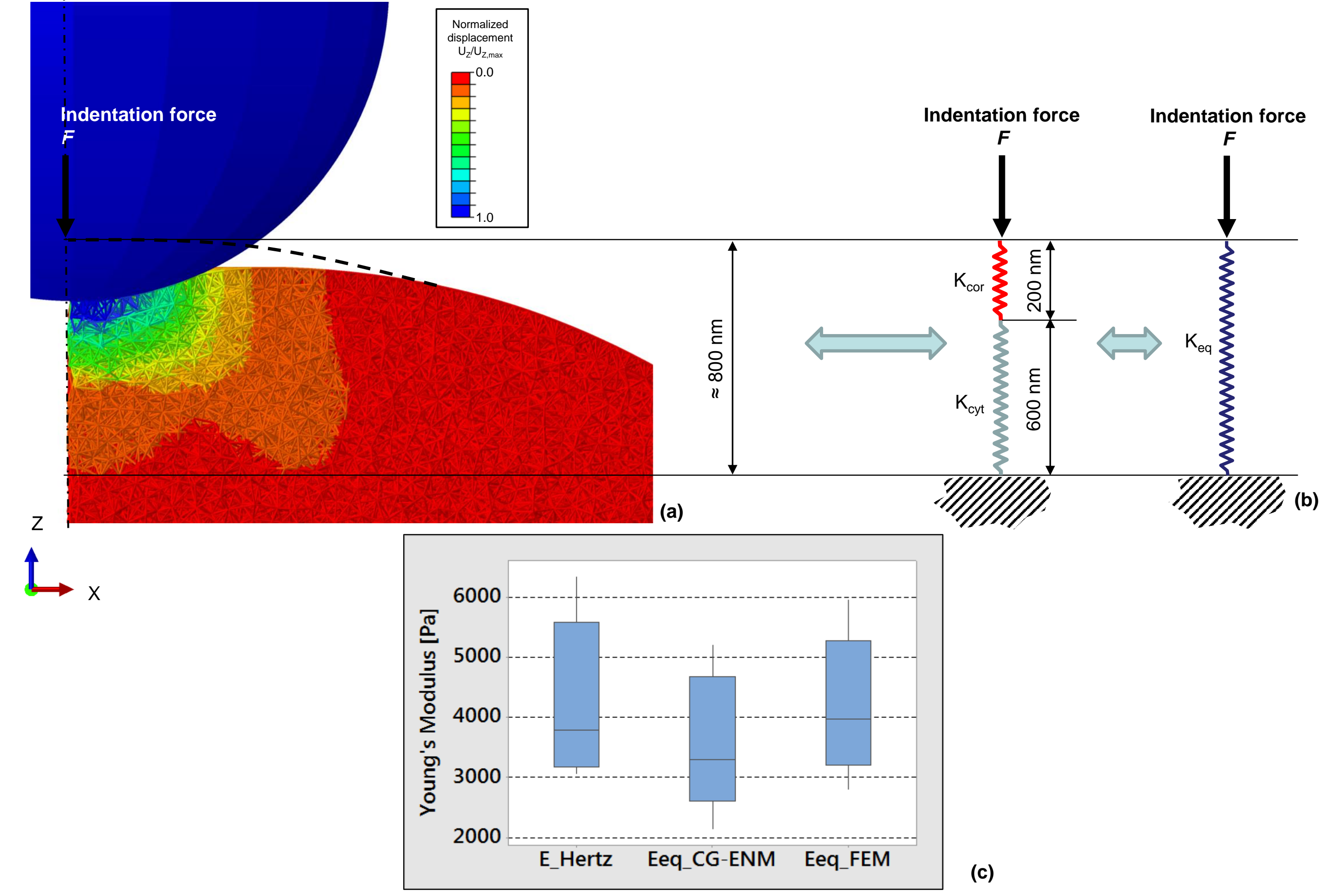

Fig. 10. (a) Normalized displacement field $U_{Z} / U_{Z m a x}$ predicted by the coarse-grained elastic network model (CG-ENM). (b) Scheme of the AFM tip-cell contact adopted to determine the equivalent Young's modulus. (c) Box plot of the equivalent Young's moduli compared with the Young's moduli computed via the Hertz contact theory. 
schematized, according to a previous study [55], with a force acting on two springs disposed in series (Figure 10(b)). Other semi-empirical approaches have been recently proposed to describe the mechanics of the double layer on which the nanoindenter acts [56-58]. The first spring, having a length $L_{c o r}=200 \mathrm{~nm}$ (i.e. the thickness of the cell cortex), a cross section $A_{\text {cor }}$ and an elastic constant $\mathrm{K}_{\mathrm{cor}}=\frac{\mathrm{E}_{\mathrm{cor}} \cdot A_{\text {cor }}}{L_{\text {cor }}}$, is representative of the elastic response of the cell cortex; the second spring, having a length $L_{c y t}=600 \mathrm{~nm}$ (i.e. the height of the cytoskeletal region where non-zero stress values act during the $200 \mathrm{~nm}$ nanoindentation), a cross section $A_{c y t}$ and an elastic constant $\mathrm{K}_{\mathrm{cyt}}=\frac{\mathrm{E}_{c y t} \cdot A_{c y t}}{L_{c y t}}$, is representative of the elastic response of the cytoskeleton. Applying the classical relationships for springs disposed in series and following the hypothesis that $A_{c o r}=A_{c y t}$ it can be easily demonstrated that the equivalent Young's modulus $\mathrm{E}_{\mathrm{eq}}$ of the spring that describes the elastic response of the two springs having $\mathrm{K}_{\text {cor }}$ and $\mathrm{K}_{\text {cyt }}$ as stiffnesses, can be expressed as:

$$
\mathrm{E}_{\mathrm{eq}}=\left(L_{c o r}+L_{c y t}\right) \cdot \frac{\mathrm{E}_{\mathrm{cor}} \cdot \mathrm{E}_{\mathrm{cyt}}}{\mathrm{E}_{\mathrm{cyt}} \cdot L_{c o r}+\mathrm{E}_{\mathrm{cor}} \cdot L_{c y t}}
$$

Applying the Eq. (2), for each cell sample, and setting the values $E_{\text {cor }}$ and $E_{\text {cyt }}$ obtained with both, the coarse-grained elastic network model (CG-ENM) and the FEM (Table 3), we determined the equivalent Young's moduli and compared them with those computed via the Hertz contact theory (Fig. 10(c)). To make statistical inferences, we started to enquire whether data followed a normal distribution. We used the Shapiro-Wilk normality test, AS R94 algorithm, on all Young's moduli sets (i.e. the set of equivalent Young's moduli computed for coarse-grained elastic network model, the set of equivalent Young's moduli computed for FEM and the set of Young's moduli computed via the Hertz contact theory). All the three sets passed the normality test $\left(\mathrm{E}_{\mathrm{Hertz}} \mathrm{W}=0.886, p\right.$-value $=0.155$; Eeq_CG-ENM: $\mathrm{W}=0.914, \quad p$-value $=0.312 ; \quad \mathrm{E}_{\text {eq_FEM: }} \mathrm{W}=0.917, \quad p$-value $\left.=0.335\right)$. To evaluate the homoscedasticity, we applied the Levene test, that revealed that samples were homoscedastic $(\mathrm{F}(2$, $27)=0.467, p$-value $=0.632$ ). Given these preliminary results, we performed ANOVA to compare the 
three Young's moduli distributions. It revealed that there is not a statistically significant difference

494 between the distributions of the equivalent Young's moduli and the Young's moduli obtained with the Hertz theory $(\mathrm{F}(2,27)=1.149$, -value $=0.332)$.

For all samples, the $R^{2}$ values were close to 1 (Table 2), which means that the assumptions made on discretization topology (refined Delaunay algorithm for tetrahedrons and an adequate high spring density for the considered deformations) and on spring behavior (linear elastic) were reasonably correct for this analysis. $R^{2}$ values computed for the FEM are slightly higher than those obtained through the coarse-grained elastic network modelling. However, it is worthy to note that the correlation coefficient computed with the FEM method derives from an optimization process performed by managing four design variables, $\mu_{8 \text { chain }}$ for cytoskeleton and cortex, and $\lambda_{L}$ for cytoskeleton and cortex) while that computed with the coarse-grained elastic network model derives from an optimization process including only two design variables $\left(E_{l o c, c o r}\right.$ and $\left.E_{l o c, c y t}\right)$. In other words, we can conclude that a 2-parameter model (coarse-grained elastic network model) provided a reasonably good and fast approximation of the results of a 4-parameter model (FEM, Arruda-Boyce). An articulated model of the cell was introduced and described with a reasonable but arbitrary number of design hypotheses. Preliminary analyses were conducted to evaluate the impact of these hypotheses on the obtained results. For instance, the quarter model of the cell was hypothesized to include nine stress fibers. We evaluated how the cell mechanical properties change for a variable number of stress fibers: fifteen, nine, four, one, or for the case of stress fibers absent. Fig. 11 shows, for instance, the force-indentation curves obtained for the same material properties (i.e E Eloc,cor,optim and 513 Eloc,cyt,optim computed for cell sample 9, Table 1) and for different numbers of stress fibers. It can be 514 seen that reducing the number of stress fibers leads, for the same nanoindentation depth, to increasing 515 values of force that the cell opposes to the AFM nanoindenter. This result can be justified with the "qualitative" argument that the stress fibers create a tensile stress state on the cellular region in contact with the AFM tip that favors the tip penetration into the cell. As the number of stress fibers decreases, the entity of the tensile stress acting on the cell decreases too and consequently the nanoindenter 
15 stress fibers



4 stress fibers



9 stress fibers

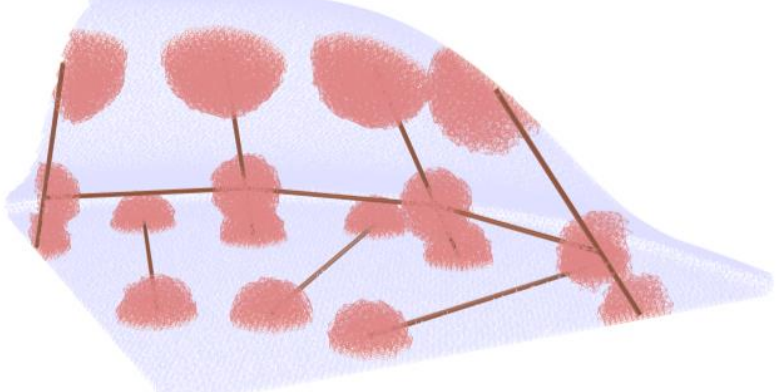

1 stress fiber

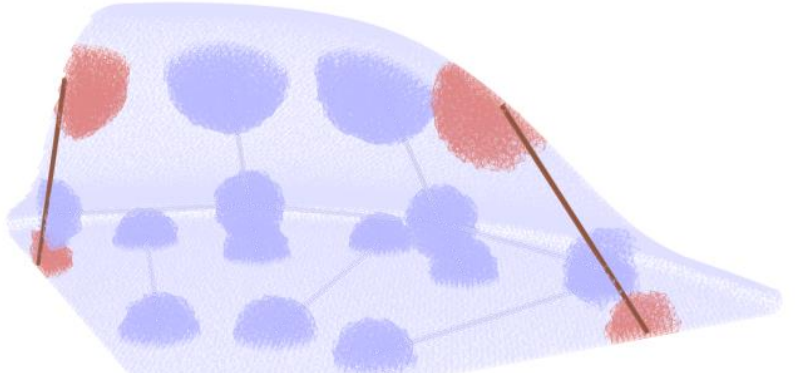

stress fibers absent

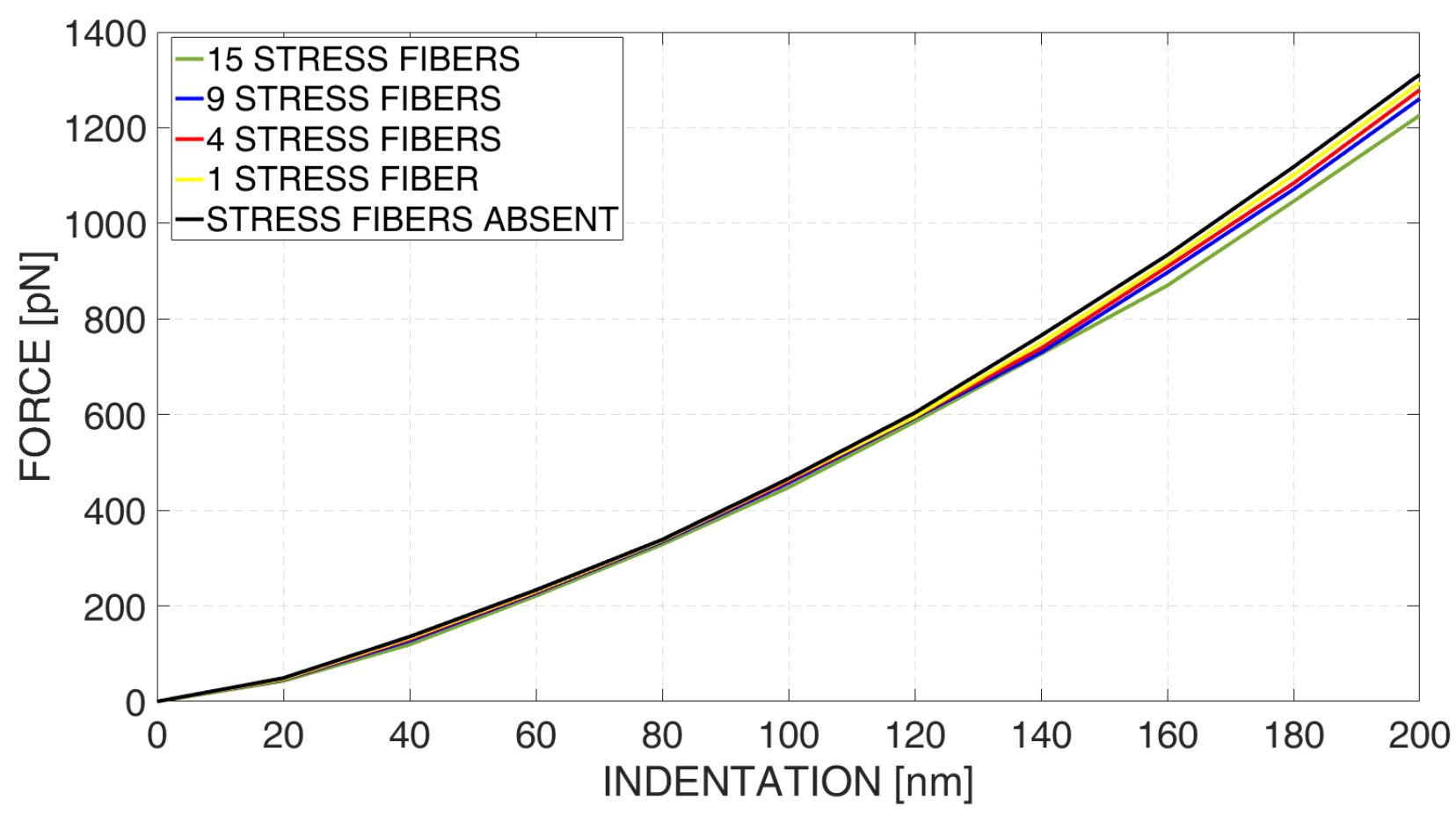

Fig. 11. Force-indentation curves computed by the model for the same material properties (i.e. $\mathrm{E}_{\text {loc,cor,optim }}$ and $\mathrm{E}_{\text {loc,cyt,optim }}$ of sample 9) and for a variable number of stress fibers. The activated (in red) and the disabled (blue) stress fibers simulated in the model are shown in the figures above the diagram. 
experiences an increasing reaction force that the cell opposes to its penetration. The global material 520 properties predicted via the proposed coarse-grained elastic network model decrease for decreasing 521 numbers of stress fibers. Table 4 lists, for instance, $E_{\text {cyt }}$ and $E_{\text {cor }}$ computed for the cell sample 9. 522 However, the percent difference - with respect to the model including nine stress fibers - never 523 exceeds $4.03 \%$ in the case of the cytoskeleton and $1.00 \%$ in the case of the cortex. These results lead us to conclude that, in view of a simpler description of the cell, a sort of reasonable result can be achieved even without stress fibers. However, the model should consider the effect of stress fibers especially in the case of cells including a large number of them. numbers of stress fibers.

\begin{tabular}{c||cc||c||c}
\hline & Optimal global properties & $\begin{array}{c}\text { Percent difference } \\
\text { cytoskeleton }\end{array}$ & $\begin{array}{c}\text { Percent } \\
\text { difference cortex }\end{array}$ \\
\hline $\begin{array}{c}\text { \# of stress } \\
\text { fibers }\end{array}$ & $E_{\text {cyt }}[\mathrm{Pa}]$ & $E_{\text {cor }}[\mathrm{Pa}]$ & {$[\%]$} & \\
\hline 15 & 2594 & 8759 & 2.65 & -0.97 \\
9 & 2527 & 8845 & - & - \\
4 & 2497 & 8815 & -1.18 & -0.34 \\
1 & 2448 & 8770 & -3.13 & -0.85 \\
0 & 2425 & 8756 & -4.03 & -1.00 \\
\hline
\end{tabular}

531 value, according to previous studies [11,59] was set one order of magnitude higher than the average 532 stiffness of the cytoskeleton. Nevertheless, recent literature has pointed out that this is not necessarily 533 the case [51]. To evaluate the effect of the hypothesized nucleus material properties on the predicted 534 values of $\mathrm{E}_{\mathrm{cyt}}$ and $\mathrm{E}_{\mathrm{cor}}$, preliminary analyses were carried out with different values of $E_{\text {loc,nuc. }}$ Figure 53512 shows, for instance, the force-indentation curves obtained implementing Eloc,cyt,optim $_{\text {and }} \mathrm{E}_{\text {loc,cor,optim }}$ 536 predicted for the cell sample 9 and three different values of the nucleus material properties $E_{l o c, n u c}$ : $537-E_{l o c, n u c}$ with the same order of magnitude as the average stiffness of the cytoskeleton; 


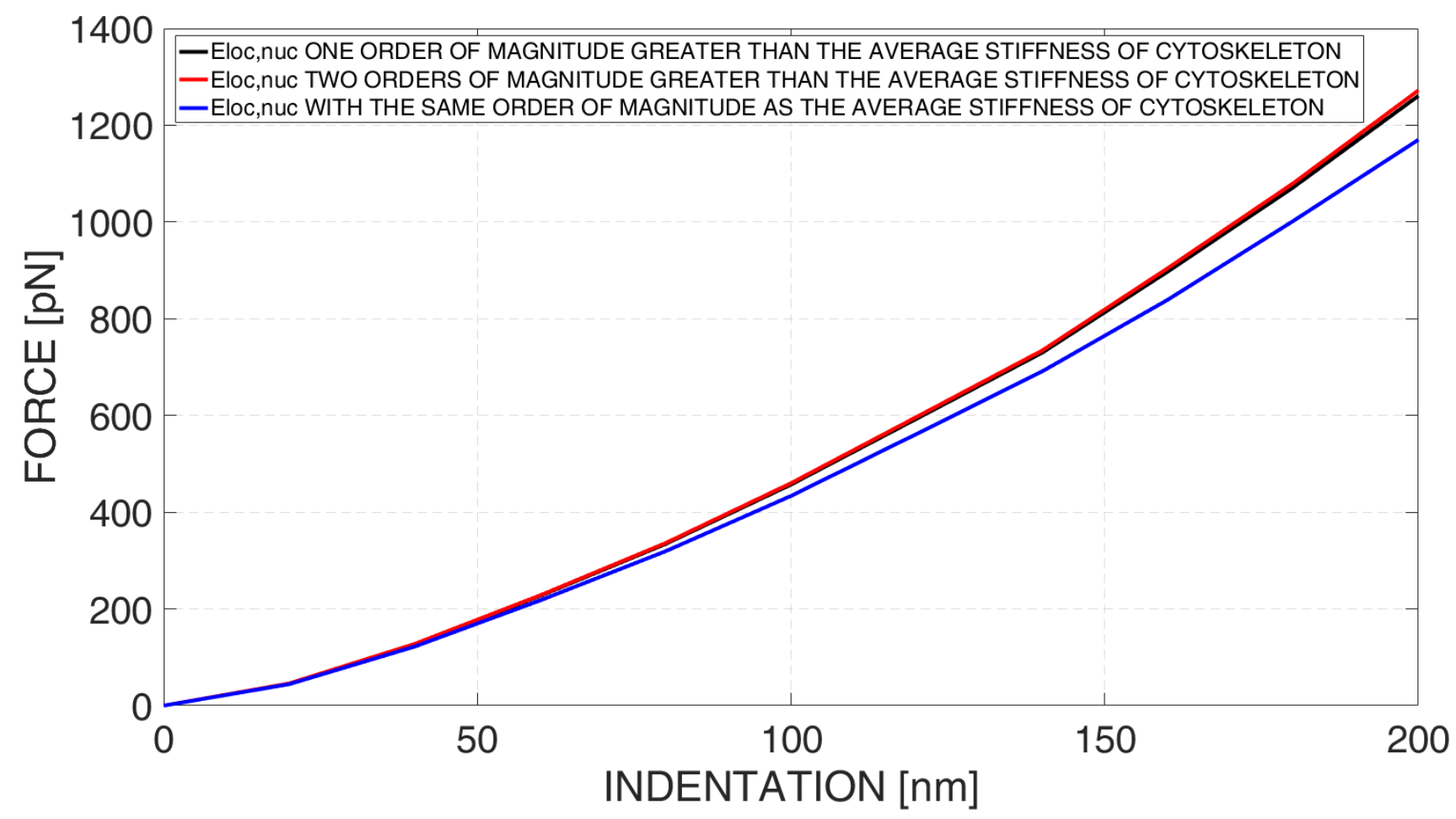

Fig. 12. Force-indentation curves computed by the model for the same material properties (i.e. $\mathrm{E}_{\mathrm{loc}, \mathrm{cor}, \mathrm{optim}}$ and $\mathrm{E}_{\mathrm{loc}, \mathrm{cyt}, \mathrm{optim}}$ computed for sample 9) and for variable values of $E_{l o c, \text { nuc }}$. 
538 - Eloc,nuc one order of magnitude higher than the average stiffness of the cytoskeleton (design

539 hypothesis);

$540-E_{l o c, n u c}$ two orders of magnitude higher the average stiffness of the cytoskeleton.

541 The force-indentation curves obtained with $E_{l o c, n u c}$ with one or two orders of magnitude higher than

542 the average stiffness of cytoskeleton are practically overlapped. Larger differences can be seen

543 instead, in the case of $E_{l o c, n u c}$ with the same order of magnitude as the average stiffness of the

544 cytoskeleton. The global material properties predicted with the proposed coarse-grained elastic 545 network model increase for decreasing values of $E_{l o c, n u c}$. Table 5 lists, for instance, the values of $E_{c y t}$ 546 and $\mathrm{E}_{\text {cor }}$ predicted by the optimization algorithm for the three values of $E_{l o c, \text { nuc }}$ as well as the percent 547 difference - computed with respect to the model with $E_{l o c, n u c}$ one order of magnitude higher than the 548 average stiffness of the cytoskeleton - of the predicted values of $\mathrm{E}_{\text {cyt }}$ and $\mathrm{E}_{\mathrm{cor}}$. It can be seen that the 549 highest value of this difference was predicted in the case of the cytoskeleton and does not exceed $5505.18 \%$.

551 Table 5. Global material properties computed for the cell sample 9, for different nucleus material properties $E_{l o c, n u c}$

\begin{tabular}{c||cc||c||c}
\hline & Optimal global properties & $\begin{array}{c}\text { Percent difference } \\
\text { cytoskeleton }\end{array}$ & $\begin{array}{c}\text { Percent } \\
\text { difference cortex }\end{array}$ \\
\hline$E_{\text {loc,nuc }}$ & $E_{\text {cyt }}[\mathrm{Pa}]$ & $\mathrm{E}_{\text {cor }}[\mathrm{Pa}]$ & {$[\%]$} & - \\
\hline $\begin{array}{c}\text { One order of } \\
\text { magnitude higher } \\
\text { (design hypothesis) } \\
\text { Two orders of } \\
\text { magnitude higher } \\
\text { Same order of } \\
\text { magnitude }\end{array}$ & 2527 & 8845 & - & -0.13 \\
\hline
\end{tabular}

The global material properties were computed by implementing a monodimensional twin 555 model with a cylindrical shape. It would be very interesting knowing how the utilized geometry 556 affects the predicted material properties. To investigate this issue, two further (in addition to the main 
one) monodimensional twin models were built (Fig. 13(a)) with the same aspect ratio $5: 1$ and having volumes equal to $\pm 10 \%$ of the original volume. Interestingly, implementing $E_{\text {loc,cyt,optim }}$ and Eloc,cor,optim $_{\text {lim }}$ predicted for the cell sample 9 and subjecting the models to a tensile test, the obtained stress/strain curves were very close one each other (Fig. 13(b) and (c)). The global material properties predicted with these two monodimensional twin models differ - with respect to the original monodimensional twin model - by very small quantities never exceeding, in absolute value, $0.32 \%$ (Table 6).

Table 6. Global material properties computed for the cell sample 9, for different geometries of the

The most interesting feature of the proposed workflow is the intrinsic compliance of the spring network, due to the missing constraints for spring rotations at nodes. This is ensured by modelling the spring connections as internal spherical hinges, thus decoupling every pair of springs and not allowing bending moment transmission, but only axial forces. From a physical point of view, such a model can be regarded as an attempt to a micromechanical representation of weak and non-directional bonds in biological materials [30]. The associated mathematical description can generally consist in a series of simply linear systems in the form $\mathrm{Ax}=\mathrm{B}$ for solving the static problem of a single incremental deformation, thus leading to a stable and fast formulation of the whole static problem of large deformation. Furthermore, it is not necessary to perform numerical interpolation on a continuum volumetric domain to compute expressions for displacement, deformation and other field variables as in finite element analysis. To demonstrate these features, as a proof of large deformation capability 


\section{diameter $\varnothing 13.52$ micron} height 67.58 micron

\section{VOLUME $=V$}

(a)
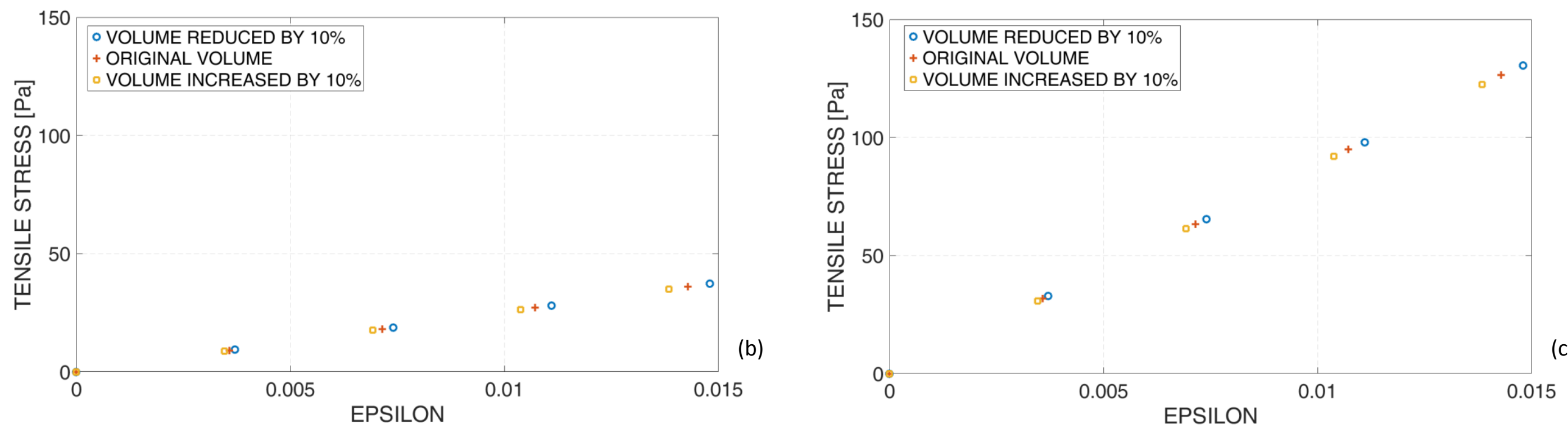

Fig. 13. (a) Monodimensional twin models utilized to assess the effect of the geometry on the predicted material properties. Stress-strain curves obtained with the models with different geometry implementing $\mathrm{E}_{\mathrm{loc}, \text { cyt,optim }}$ (b) and $\mathrm{E}_{\mathrm{loc}, \text { cor,optim }}$ (c) computed for cell sample 9 (Table 1). Only markers are represented (and not lines) to better visualize the very small differences between the three models. 
in Fig. 14 (a) and (c) are presented undeformed and related deformed configurations for two

579 monodimensional twin models built, one with the FEM method the other with a spring network. Both 580 the models were hypothesized to be linear elastic. A flat base of the cylinder was constrained while a 581 sufficiently large displacement was imposed to the other flat base via a rigid plate to understand until 582 which extent the two models guarantee numerical convergence. In the FEM model, the value of the 583 material properties implemented is $E_{c o r}$ determined for sample 1, whereas in the spring network model

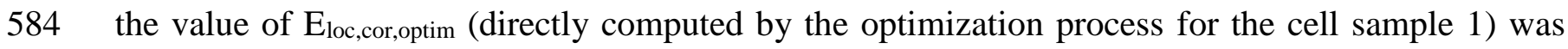
585 utilized. Interestingly, the spring network showed larger deformation than FEM until reaching the numerical non-convergence condition and thus the end of the analysis (Fig. 14b). The two curves presented the same tangent and were practically overlapped for small values of displacement but tended to diverge, due to the intrinsic different characteristics of the two models, for very large values of displacement.

Another remarkable result to point out is the comparison between the computation times necessary to complete the simulation. Generally, the duration of each analysis on a spring network model is about $1 / 3$ of the time necessary to complete a FEM analysis with a comparable density of elements.

\section{Conclusions}

This paper describes a physical/mathematical method for extracting Young's moduli of a 597 deformable solid structure. The study focused on nanoindented human mesenchymal stem cells and 598 reproduced through a coarse-grained elastic network a real AFM experiment. An optimization 599 algorithm iterated a loop until a satisfactory fit between real and simulated force-indentation curves 600 was reached. The topological and behavioral characteristics of the simulated cells were then 601 transferred to a monodimensional twin model and the Young's moduli for subcellular components 602 were extracted through a virtual tensile test. 

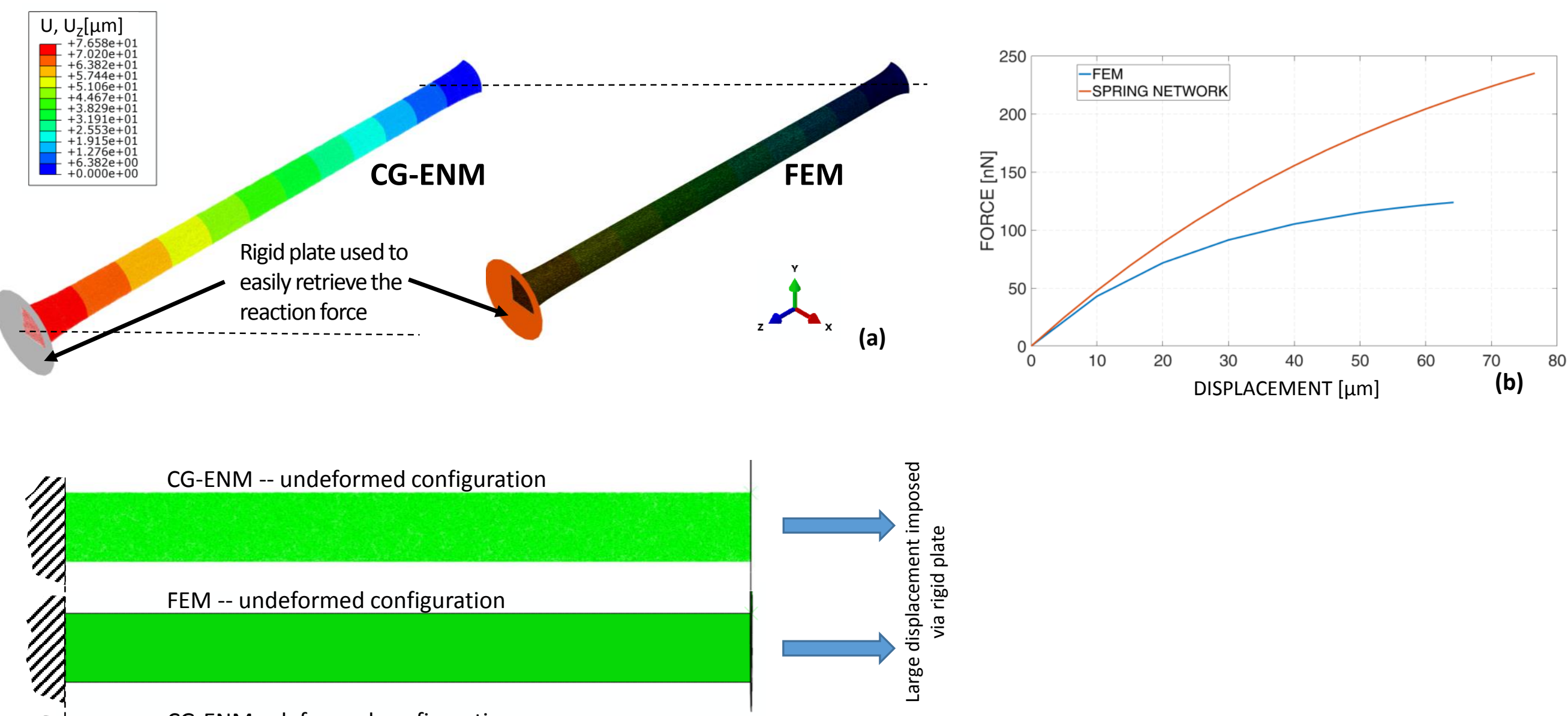

CG-ENM-- deformed configuration

Fig. 14. (a) 3D view of virtual tensile tests performed through coarse-grained elastic network model (CG-ENM) and FEM. (b) An example of forcedisplacement curves obtained with CG-ENM compared to that obtained with FEM. (c) Comparison between undeformed and deformed configurations of CG-ENM and FEM models. 
The resulting Young's moduli showed good agreement with the corresponding moduli 604 resulting from a previous study, performed using the Finite Element Method, and also presented the 605 same order of magnitude as the elastic moduli predicted by hertzian contact theory and related 606 literature.

The proposed approach can be considered as a useful and fast tool to study the mechanical 608 behavior of biological soft materials. As a general rule, in a coarse-grained elastic network model, 609 the time required to complete the simulation is about $33 \%$ of the time required to run the same 610 simulation with a FEM model presenting the same discretization density for the elements. 611 Remarkable numerical stability and computational speed make this method comparable to a 612 traditional FEM analysis and even preferable in the case of large displacements and deformations of 613 soft materials.

\section{Declaration of Competing Interest}

The authors have declared that no competing interests exist.

Ethics statement

Bone marrow from healthy donors for allogeneic transplantation was taken after written consent using guidelines approved by the Ethic Committee on the use of Human subjects at the Heidelberg University.

\section{Acknowledgements}

The support given by Dr. Patrick Horn (Department of Medicine V, Heidelberg University), 
626 Union's Framework Program for Research and Innovation Horizon 2020 (2014-2020) under the 627 Marie Sklodowska-Curie Grant Agreement No. 658334 for the funding received.

\section{References}

630 [1] D.A. Lee, M.M. Knight, J.J. Campbell, D.L. Bader, Stem cell mechanobiology, J. Cell. Biochem. 112 (2011) 1-9. doi:10.1002/jcb.22758.

[2] M.C.H. Van der Meulen, R. Huiskes, Why mechanobiology? A survey article, J. Biomech. 35 (2002) 401-414. doi:10.1016/S0021-9290(01)00184-1.

[3] G. Bao, S. Suresh, Cell and molecular mechanics of biological materials, Nat. Mater. 2 (2003) 715-725. www.nature.com/naturematerials.

[4] C.T. Lim, E.H. Zhou, S.T. Quek, Mechanical models for living cells - A review, J. Biomech. 39 (2006) 195-216. doi:10.1016/j.jbiomech.2004.12.008.

638 [5] J.D. Humphrey, Vascular adaptation and mechanical homeostasis at tissue, cellular, and sub639 cellular levels, Cell Biochem. Biophys. 50 (2008) 53-78. doi:10.1007/s12013-007-9002-3.

[6] S. Cantore, V. Crincoli, A. Boccaccio, A.E. Uva, M. Fiorentino, G. Monno, P. Bollero, C.

[7] A. Boccaccio, M. Fiorentino, A.E. Uva, L.N. Laghetti, G. Monno, Rhombicuboctahedron unit cell based scaffolds for bone regeneration: geometry optimization with a mechanobiology driven algorithm, Mater. Sci. Eng. C. 83 (2018) 51-66. doi:10.1016/j.msec.2017.09.004. Derla, F. Fabiano, A. Ballini, L. Santacroce, Recent Advances in Endocrine, Metabolic and Immune Disorders: Mesenchymal Stem Cells (MSCs) and Engineered Scaffolds, Endocrine, Metab. Immune Disord. - Drug Targets. $18 \quad$ (2018) 466-469. doi:10.2174/1871530318666180423102905.

[8] Ó.L. Rodríguez-Montaño, C.J. Cortés-Rodríguez, F. Naddeo, A.E. Uva, M. Fiorentino, A. Naddeo, N. Cappetti, M. Gattullo, G. Monno, A. Boccaccio, Irregular Load Adapted Scaffold Optimization: A Computational Framework Based on Mechanobiological Criteria, ACS 
Biomater. Sci. Eng. 5 (2019) 5392-5411. doi:10.1021/acsbiomaterials.9b01023.

652 [9] M.J. Rosenbluth, W.A. Lam, D.A. Fletcher, Force microscopy of nonadherent cells: A comparison of leukemia cell deformability, Biophys. J. 90 (2006) 2994-3003. doi:10.1529/biophysj.105.067496.

[10] M. Murakoshi, N. Yoshida, K. Iida, S. Kumano, T. Kobayashi, H. Wada, Local mechanical properties of mouse outer hair cells: Atomic force microscopic study, Auris Nasus Larynx. 33

[11] A. Boccaccio, A.E. Uva, M. Papi, M. Fiorentino, M. De Spirito, G. Monno, Nanoindentation

[14] P.K.V. Babu, M. Radmacher, Mechanics of brain tissues studied by atomic force microscopy: A perspective, Front. Neurosci. 13 (2019) 1-9. doi:10.3389/fnins.2019.00600.

[15] P. Duan, R. Toumpaniari, S. Partridge, M.A. Birch, P.G. Genever, S.J. Bull, K.W. Dalgarno, A.W. McCaskie, J. Chen, How cell culture conditions affect the microstructure and nanomechanical properties of extracellular matrix formed by immortalized human mesenchymal stem cells: An experimental and modelling study, Mater. Sci. Eng. C. 89 (2018) 149-159. doi:10.1016/j.msec.2018.03.027.

[16] A. Herrera, J. Hellwig, H. Leemhuis, R. von Klitzing, I. Heschel, G.N. Duda, A. Petersen, 
From macroscopic mechanics to cell-effective stiffness within highly aligned macroporous collagen scaffolds, Mater. Sci. Eng. C. 103 (2019) 109760. doi:10.1016/j.msec.2019.109760.

[17] O. Piétrement, E. Jallot, AFM mechanical mapping at the interface between a bioactive glass coating and bone, Nanotechnology. 13 (2002) 18-22. doi:10.1088/0957-4484/13/1/304.

[19] R. Vargas-Pinto, H. Gong, A. Vahabikashi, M. Johnson, The effect of the endothelial cell cortex on atomic force microscopy measurements, Biophys. J. 105 (2013) 300-309. doi:10.1016/j.bpj.2013.05.034.

[20] K.K. Liu, Deformation behaviour of soft particles: A review, J. Phys. D. Appl. Phys. 39 (2006). doi:10.1088/0022-3727/39/11/R01.

[21] A. Boccaccio, L. Lamberti, M. Papi, M. De Spirito, C. Pappalettere, Effect of AFM probe geometry on visco-hyperelastic characterization of soft materials, Nanotechnology. 26 (2015) 325701. doi:10.1088/0957-4484/26/32/325701.

[22] A. Boccaccio, M. Papi, M. De Spirito, L. Lamberti, C. Pappalettere, Effect of the residual stress on soft sample nanoindentation, Appl. Phys. Lett. 102 (2013) 1-5. doi:10.1063/1.4801428.

[23] D.C. Lin, D.I. Shreiber, E.K. Dimitriadis, F. Horkay, Spherical indentation of soft matter beyond the Hertzian regime: Numerical and experimental validation of hyperelastic models, Biomech. Model. Mechanobiol. 8 (2009) 345-358. doi:10.1007/s10237-008-0139-9.

[24] E. Migliorini, E.A. Cavalcanti-Adam, A.E. Uva, M. Fiorentino, M. Gattullo, V.M. Manghisi, L. Vaiani, A. Boccaccio, Nanoindentation of Mesenchymal Stem Cells using Atomic Force Microscopy: Effect of Adhesive Cell-Substrate Structures, Submitted.

[25] M.O. Steinhauser, S. Hiermaier, A review of computational methods in materials science: Examples from shock-wave and polymer physics, Int. J. Mol. Sci. 10 (2009) 5135-5216. 
doi:10.3390/ijms 10125135.

704 [26] R. Huiskes, E.Y.S. Chao, A survey of finite element analysis in orthopedic biomechanics: the first decade, J. Biomech. 16 (1983) 385-409.

[27] O.C. Zienkiewicz, P.B. Morice, The finite element method in engineering science, McGrawHill London, 1971.

[28] V. Rajagopal, W.R. Holmes, P.V.S. Lee, Computational modeling of single-cell mechanics 709 and cytoskeletal mechanobiology, Wiley Interdiscip. Rev. Syst. Biol. Med. 10 (2018) 5-7.

[29] T. Ye, N. Phan-Thien, C.T. Lim, Particle-based simulations of red blood cells-A review, J. Biomech. 49 (2016) 2255-2266. doi:10.1016/j.jbiomech.2015.11.050. doi:10.1002/wsbm.1407.

[30] M. Vassaux, J.L. Milan, Stem cell mechanical behaviour modelling: substrate's curvature influence during adhesion, Biomech. Model. Mechanobiol. 16 (2017) 1295-1308. doi:10.1007/s 10237-017-0888-4.

[31] H.I. Ingólfsson, C.A. Lopez, J.J. Uusitalo, D.H. de Jong, S.M. Gopal, X. Periole, S.J. Marrink, The power of coarse graining in biomolecular simulations, Wiley Interdiscip. Rev. Comput. Mol. Sci. 4 (2014) 225-248. doi:10.1002/wcms.1169.

[32] Y. Togashi, H. Flechsig, Coarse-grained protein dynamics studies using elastic network models, Int. J. Mol. Sci. 19 (2018). doi:10.3390/ijms19123899.

[33] M. Kot, H. Nagahashi, P. Szymczak, Elastic moduli of simple mass spring models, Vis. Comput. 31 (2015) 1339-1350. doi:10.1007/s00371-014-1015-5.

[34] W. Wagner, P. Horn, M. Castoldi, A. Diehlmann, S. Bork, R. Saffrich, V. Benes, J. Blake, S. Pfister, V. Eckstein, A.D. Ho, Replicative senescence of mesenchymal stem cells: A continuous and organized process, PLoS One. 3 (2008). doi:10.1371/journal.pone.0002213.

[35] L. Blanchoin, R. Boujemaa-Paterski, C. Sykes, J. Plastino, Actin dynamics, architecture, and mechanics in cell motility, Physiol. Rev. 94 (2014) 235-263. doi:10.1152/physrev.00018.2013. 
[36] R. Oria, T. Wiegand, J. Escribano, A. Elosegui-Artola, J.J. Uriarte, C. Moreno-Pulido, I. Platzman, P. Delcanale, L. Albertazzi, D. Navajas, X. Trepat, J.M. García-Aznar, E.A. Cavalcanti-Adam, P. Roca-Cusachs, Force loading explains spatial sensing of ligands by cells, Nature. 552 (2017) 219-224. doi:10.1038/nature24662.

[37] Y. Liu, R. Medda, Z. Liu, K. Galior, K. Yehl, J.P. Spatz, E.A. Cavalcanti-Adam, K. Salaita, Nanoparticle tension probes patterned at the nanoscale: Impact of integrin clustering on force transmission, Nano Lett. 14 (2014) 5539-5546. doi:10.1021/nl501912g.

[38] A.G.F. De Beer, E.A. Cavalcanti-Adam, G. Majer, M. Lopez-García, H. Kessler, J.P. Spatz, Force-induced destabilization of focal adhesions at defined integrin spacings on nanostructured surfaces, Phys. Rev. E - Stat. Nonlinear, Soft Matter Phys. 81 (2010) 1-7. doi:10.1103/PhysRevE.81.051914.

[39] A. Livne, B. Geiger, The inner workings of stress fibers - From contractile machinery to focal adhesions and back, J. Cell Sci. 129 (2016) 1293-1304. doi:10.1242/jcs.180927.

[40] S. Deguchi, T. Ohashi, M. Sato, Tensile properties of single stress fibers isolated from cultured vascular smooth muscle cells, J. Biomech. 39 (2006) 2603-2610. doi:10.1016/j.jbiomech.2005.08.026.

[41] M. Théry, A. Pépin, E. Dressaire, Y. Chen, M. Bornens, Cell distribution of stress fibres in response to the geometry of the adhesive environment, Cell Motil. Cytoskeleton. 63 (2006) 341-355. doi:10.1002/cm.20126.

[42] K. Burridge, C. Guilluy, Focal adhesions, stress fibers and mechanical tension, Exp. Cell Res. 343 (2016) 14-20. doi:10.1016/j.yexcr.2015.10.029.

[43] X. Su, H. Zhou, G. Bao, J. Wang, L. Liu, Q. Zheng, M. Guo, J. Zhang, Nanomorphological and mechanical reconstruction of mesenchymal stem cells during early apoptosis detected by atomic force microscopy, Biol. Open. 9 (2020). doi:10.1242/bio.048108.

[44] H. Rogge, N. Artelt, N. Endlich, K. Endlich, Automated segmentation and quantification of actin stress fibres undergoing experimentally induced changes, J. Microsc. 268 (2017) 129- 
140. doi:10.1111/jmi.12593.

[45] K. Nagayama, T. Matsumoto, Estimation of single stress fiber stiffness in cultured aortic smooth muscle cells under relaxed and contracted states: Its relation to dynamic rearrangement of stress fibers, J. Biomech. 43 (2010) 1443-1449. doi:10.1016/j.jbiomech.2010.02.007.

[46] U.S. Schwarz, N.Q. Balaban, D. Riveline, A. Bershadsky, B. Geiger, S.A. Safran, Calculation of forces at focal adhesions from elastic substrate data: The effect of localized force and the need for regularization, Biophys. J. 83 (2002) 1380-1394. doi:10.1016/S0006-3495(02)73909$\mathrm{X}$.

[47] N.Q. Balaban, U.S. Schwarz, D. Riveline, P. Goichberg, G. Tzur, I. Sabanay, D. Mahalu, S. Safran, A. Bershadsky, L. Addadi, B. Geiger, Force and focal adhesion assembly: a close relationship studied using elastic micropatterned substrates, Nat. Cell Biol. 3 (2001) 466-472. doi:10.1038/35074532.

[48] K. Burridge, E.S. Wittchen, The tension mounts: Stress fibers as force-generating mechanotransducers, J. Cell Biol. 200 (2013) 9-19. doi:10.1083/jcb.201210090.

[49] E.M. Arruda, M.C. Boyce, A three-dimensional constitutive model for the large stretch behavior of rubber elastic materials, J. Mech. Phys. Solids. 41 (1993) 389-412. doi:10.1016/0022-5096(93)90013-6.

[50] J.W. Harding, I.N. Sneddon, The elastic stresses produced by the indentation of the plane surface of a semi-infinite elastic solid by a rigid punch, Math. Proc. Cambridge Philos. Soc. 41 (1945) 16-26. doi:DOI: 10.1017/S0305004100022325.

[51] T. Fischer, A. Hayn, C.T. Mierke, Effect of Nuclear Stiffness on Cell Mechanics and Migration of Human Breast Cancer Cells, Front. Cell Dev. Biol. 8 (2020) 1-18. doi:10.3389/fcell.2020.00393.

[52] Q. Chen, P. Xiao, J.N. Chen, J.Y. Cai, X.F. Cai, H. Ding, Y.L. Pan, AFM studies of cellular mechanics during osteogenic differentiation of human amniotic fluid-derived stem cells, Anal. Sci. 26 (2010) 1033-1037. doi:10.2116/analsci.26.1033. 
[53] R. Kiss, H. Bock, S. Pells, E. Canetta, A.K. Adya, A.J. Moore, P. De Sousa, N.A. Willoughby, Elasticity of human embryonic stem cells as determined by atomic force microscopy, J. Biomech. Eng. 133 (2011) 1-10. doi:10.1115/1.4005286.

[54] X. Liang, X. Shi, S. Ostrovidov, H. Wu, K. Nakajima, Probing stem cell differentiation using atomic force microscopy, Appl. Surf. Sci. $366 \quad$ (2016) 254-259. doi:10.1016/j.apsusc.2016.01.082.

[55] P.M. Ogar, A.S. Kozhevnikov, V.S. Kushnarev, Contact characteristics of a sphere with a layered elastic half-space, IOP Conf. Ser. Mater. Sci. Eng. 709 (2020). doi:10.1088/1757899X/709/3/033111.

[56] I. Lüchtefeld, A. Bartolozzi, J. Mejía Morales, O. Dobre, M. Basso, T. Zambelli, M. Vassalli, Elasticity spectra as a tool to investigate actin cortex mechanics, J. Nanobiotechnology. 18 (2020) 1-11. doi:10.1186/s12951-020-00706-2.

[57] B.L. Doss, K. Rahmani Eliato, K. hui Lin, R. Ros, Quantitative mechanical analysis of indentations on layered, soft elastic materials, Soft Matter. 15 (2019) 1776-1784. doi:10.1039/c8sm02121j.

[58] J. Rheinlaender, A. Dimitracopoulos, B. Wallmeyer, N.M. Kronenberg, K.J. Chalut, M.C. Gather, T. Betz, G. Charras, K. Franze, Cortical cell stiffness is independent of substrate mechanics, Nat. Mater. 19 (2020) 1019-1025. doi:10.1038/s41563-020-0684-x.

[59] S. Deguchi, M. Yano, K. Hashimoto, H. Fukamachi, S. Washio, K. Tsujioka, Assessment of the mechanical properties of the nucleus inside a spherical endothelial cell based on microtensile testing, J. Mech. Mater. Struct. 2 (2007) 1087-1102. doi:10.2140/jomms.2007.2.1087. 
Fig. 1. Scheme of the workflow implemented to extract the stem cells material properties.

Fig. 2. Exploiting the symmetry properties of the entire cell geometry (a), just one-quarter model was considered (b). (c) Principal dimensions in micrometers of the model used in the study.

Fig. 3. The CAD model (a) generated in Rhinoceros was given in input to the open source meshing 813 software Gmsh (version 4.5.2) where a tetrahedral volumetric mesh was generated (b). The 814 tetrahedral mesh was then converted into a spring network model (c). To generate the quarter model, 815 a portion of the whole cell larger than the quarter portion was initially considered (a-c). A clipping 816 procedure was finally executed (d) to isolate the portion of the spring network model exactly 817 corresponding to the quarter of the cell. The aligned edges of tetrahedrons shown in (b) have been 818 obtained at the intersection of the planes delimiting the model outer surface. The clipping procedure 819 successively carried out, allows having not aligned edges (and hence not aligned springs) on the axis 820 along which the nanoindenter acts and consequently, an isotropic behavior of the model.

822 Fig. 4. Spring groups defined in the model.

Fig. 5. Boundary and loading conditions imposed to the cell in the simulation environment. For the sake of clarity only the forces acting on the visible tensioning groups are indicated and not those acting on the internal ones. 
828 Fig. 6. The overall force in every group (a) was distributed as $25 \%$ in an outer shell and $75 \%$ in an 829 inner half-sphere (b), in order to realize a sort of force gradient when moving from the highly830 tensioned central region to the unloaded free elements neighboring the tensioning group.

832 Fig. 7. Scheme of the optimization algorithm implemented to determine the optimal local material 833 properties for cytoskeleton and cortex.

835 Fig. 8. (a) Simulation setup for the monodimensional twin model (dimensions in micrometers); (b) 836 Magnified cross-sectional view of the model; c) Procedure for the extraction of global Young's 837 moduli.

Fig. 9. Experimental force-indentation curves registered for the ten stem cell samples and related numerical curves predicted via the optimization algorithm. Error bars were superimposed to the average experimental force-indentation curves to show the dispersion of data at different indentation depths.

Fig. 10. (a) Normalized displacement field $U_{Z} / U_{Z \max }$ predicted by the coarse-grained elastic network model (CG-ENM). (b) Scheme of the AFM tip-cell contact adopted to determine the equivalent Young's modulus. (c) Box plot of the equivalent Young's moduli compared with the Young's moduli

849 Fig. 11. Force-indentation curves computed by the model for the same material properties (i.e.

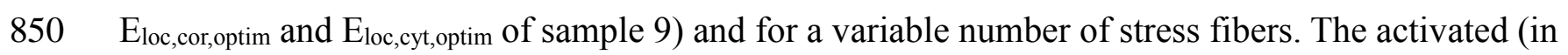
851 red) and the disabled (blue) stress fibers simulated in the model are shown in the figures above the 852 diagram. 
853 Fig. 12. Force-indentation curves computed by the model for the same material properties (i.e.

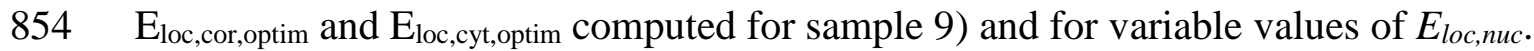

855

856 Fig. 13. (a) Monodimensional twin models utilized to assess the effect of the geometry on the 857 predicted material properties. Stress-strain curves obtained with the models with different geometry

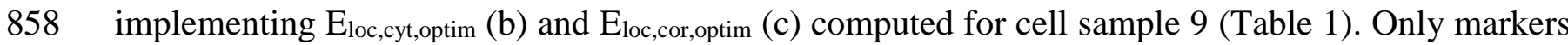
859 are represented (and not lines) to better visualize the very small differences between the three models. 860

861 Fig. 14. (a) 3D view of virtual tensile tests performed through coarse-grained elastic network model 862 (CG-ENM) and FEM. (b) An example of force-displacement curves obtained with CG-ENM 863 compared to that obtained with FEM. (c) Comparison between undeformed and deformed 864 configurations of CG-ENM and FEM models. 865 866 